\title{
Pacific
}

Journal of

Mathematics

\section{RELATIVELY MAXIMUM VOLUME RIGIDITY IN ALEXANDROV GEOMETRY}

NAN Li AND XiaOCHUN RONG 


\title{
RELATIVELY MAXIMUM VOLUME RIGIDITY IN ALEXANDROV GEOMETRY
}

\author{
NAN Li AND XIAOCHUN RonG
}

\begin{abstract}
Given compact metric spaces $X$ and $Z$ with Hausdorff dimension $n$, if there is a distance-nonincreasing onto map $f: Z \rightarrow X$, then the Hausdorff $n$ $\operatorname{volumes} \operatorname{satisfy} \operatorname{vol}(X) \leq \operatorname{vol}(Z)$. The relatively maximum volume conjecture says that if $X$ and $Z$ are both Alexandrov spaces and $\operatorname{vol}(X)=\operatorname{vol}(Z), X$ is isometric to a gluing space produced from $Z$ along its boundary $\partial Z$ and $f$ is length-preserving. We partially verify this conjecture and give a further classification for compact Alexandrov $\boldsymbol{n}$-spaces with relatively maximum volume in terms of a fixed radius and space of directions. We also give an elementary proof for a pointed version of the Bishop-Gromov relative volume comparison with rigidity in Alexandrov geometry.
\end{abstract}

\section{Introduction}

Let $Z$ be a compact metric space with Hausdorff dimension $\alpha$. Consider all compact metric spaces $X$ with Hausdorff dimension $\alpha$ such that there is a distancenonincreasing onto map $f: Z \rightarrow X$. We let "vol" denote the Hausdorff measure (or volume) in the top dimension. Then vol $X \leq \operatorname{vol} Z$. A natural question is to determine $X$ (in terms of $Z$ ) when vol $X=\operatorname{vol} Z$. We refer to this as a relatively maximum volume rigidity problem.

A possible answer to the relatively maximum volume rigidity problem is closely related to the regularity of underlying geometric and topological structures. For instance, if $Z$ and $X$ are closed Riemannian $n$-manifolds, $f$ is an isometry (see Corollary 0.2). On the other hand, taking any measure-zero subset $S$ in $Z$ (a Riemannian manifold) and identifying $S$ with a point $p \in S$, the projection map, $Z \rightarrow X=Z /(S \sim p)$, is a distance-nonincreasing onto map, and it is hopeless to have some rigidity on $Y$ in terms of $X$.

In this paper, we will study the relatively maximum volume rigidity problem in Alexandrov geometry, partly because an Alexandrov space $X$ has a "right" geometric structure for this problem (see Conjecture 0.1 below). For instance, for

Both authors are partially supported by NSF grant DMS 0805928 and by a research fund from Capital Normal University.

MSC2010: 53C21, 53C23, 53C24.

Keywords: volume, radius, Alexandrov space, rigidity, stability . 
$p \in X$, the gradient-exponential map, $g \exp _{p}: T_{p} X \rightarrow X$, becomes a distancenonincreasing map, when $T_{p} X$ is equipped with the $\kappa$-cone metric via the cosine law on the space form $S_{\kappa}^{2}$; see [Burago et al. 1992]. When taking $Z$ to be a closed $r$-ball at the vertex (for $\kappa>0, r \leq \pi /(2 \sqrt{\kappa})$ or $r=\pi / \sqrt{\kappa}$ ), the relatively volume rigidity problem (see Theorem $\mathrm{B}$ ) indeed extends the (absolutely) maximum radiusvolume rigidity theorem proved in [Grove and Petersen 1992]; see Theorem 0.3.

The recent study of Alexandrov spaces was initiated by Burago, Gromov, and Perelman [Burago et al. 1992] and has gotten a lot of attention lately. An Alexandrov space with curvature curv $\geq \kappa$ is a length metric space such that each point has a neighborhood in which the Toponogov triangle comparison holds with respect to the space form of constant curvature $\kappa$. In the rest of the paper, we will freely use basic notions on an Alexandrov space from [Burago et al. 1992] and [Petrunin 2007]; for example, the space of directions, the gradient-exponential maps, and $(n, \delta)$-strained points, among others. Let $\operatorname{Alex}^{n}(\kappa)$ denote the collection of compact Alexandrov $n$-spaces with curv $\geq \kappa$.

Note that the boundary gluing will automatically yield a distance-nonincreasing onto (projection) map, which also preserves the volume (see Examples 2.14 and 2.15). We propose the following relatively maximum volume rigidity conjecture for Alexandrov spaces.

Conjecture 0.1. Consider $Z, X \in \operatorname{Alex}^{n}(\kappa)$, and let $f: Z \rightarrow X$ be a distance-nonincreasing onto map. If $\operatorname{vol} Z=\operatorname{vol} X, X$ is isometric to a gluing space produced from $Z$ along its boundary $\partial Z$ and $f$ is length-preserving. In particular, $Z$ is isometric to $X$ if $\partial Z=\varnothing$ or if $f$ is injective.

Our goal in this paper is to partially verify Conjecture 0.1 and give a classification for the boundary gluing maps in a special case (see Theorem A, Corollary 0.2, and Theorem B).

We now begin to state the main results. Throughout this paper, $\tau(\delta)$ denotes a function in $\delta$ such that $\tau(\delta) \rightarrow 0$ as $\delta \rightarrow 0$. Our first result verifies Conjecture 0.1 for the case where $f$ preserves non- $(n, \delta)$-strained points up to an error $\tau(\delta)$. For $X \in \operatorname{Alex}^{n}(\kappa)$ and $\delta>0$, let $X^{\delta} \subseteq X$ denote the set of all $(n, \delta)$-strained points. Then a small ball centered at an $(n, \delta)$-strained point is almost isometric to an open subset in $\mathbb{R}^{n}$ [Burago et al. 1992].

Theorem A. Let $Z, X$ be Alexandrov $n$-spaces (not necessarily complete) with curvature curv $\geq \kappa$ and $\operatorname{vol} Z=\operatorname{vol} X$. Suppose that $f: Z \rightarrow X$ is a distancenonincreasing onto map such that for any $\delta>0, f^{-1}\left(X^{\delta}\right) \subseteq Z^{\tau(\delta)}$. Then $f$ is an isometry.

A point $z$ in $Z$ is called regular if the space of directions $\Sigma_{x}$ is isometric to a unit sphere. Clearly, the space $Z$ with all points regular is a topological manifold, but 
$Z$ may not be isometric to any Riemannian manifold (for example, the doubling of two flat disks). Theorem A includes the following case:

Corollary 0.2. Let $Z, X \in \operatorname{Alex}^{n}(\kappa)$ with vol $Z=\operatorname{vol} X$ and all points in $Z$ regular (for example, $Z$ is a Riemannian manifold). If $f: Z \rightarrow X$ is a distancenonincreasing onto map, $f$ is an isometry.

In Alexandrov geometry, perhaps the most natural distance-nonincreasing onto map is the gradient-exponential map $g \exp _{p}: C_{\kappa}\left(\Sigma_{p}\right) \rightarrow X, p \in X \in \operatorname{Alex}^{n}(\kappa)$, where $C_{\kappa}\left(\Sigma_{p}\right)$ denotes the tangent cone $T_{p} X$ equipped with a $\kappa$-cone metric via the cosine law in $S_{\kappa}^{2}$ [Burago et al. 1992]. Since $g \exp _{p}$ is distance-nonincreasing and preserves any $r$-ball, we immediately get the pointed version of the Bishop type volume comparison:

$$
\operatorname{vol} B_{R}(p) \leq \operatorname{vol} C_{\kappa}^{R}\left(\Sigma_{p}\right)
$$

where $C_{\kappa}^{R}\left(\Sigma_{p}\right)$ denotes the open $R$-ball in $C_{\kappa}\left(\Sigma_{p}\right)$ at the vertex $\tilde{o}$. We show that when the equality holds, $g \exp _{p}$ will satisfy the conditions in Theorem A (Lemmas 2.4 and 2.5) and thus open ball $C_{\kappa}^{R}\left(\Sigma_{p}\right)$ is isometric to $B_{R}(p)$ with respect to intrinsic metrics (see Theorem 2.1).

We prove an important case of Conjecture 0.1, which gives a classification of Alexandrov spaces with relatively maximum volume: given any $\kappa, R>0$ and $\Sigma \in$ $\operatorname{Alex}^{n-1}(1)$, let $\mathscr{A}_{\kappa}^{R}(\Sigma)$ be the collection of Alexandrov $n$-spaces $X \ni p$ satisfying

$$
\operatorname{curv} \geq \kappa, \quad X=\bar{B}_{R}(p), \quad \Sigma_{p}=\Sigma .
$$

Then vol $X \leq \operatorname{vol} C_{\kappa}^{R}(\Sigma)=v(\Sigma, \kappa, R)$. When vol $X=v(\Sigma, \kappa, R)$, we say that $X$ has the relatively maximum volume.

Theorem $\mathbf{B}$ (relatively maximum volume rigidity). Let $X \in \mathscr{A}_{\kappa}^{R}(\Sigma)$ such that $\operatorname{vol} X=v(\Sigma, \kappa, R)$. Then $X$ is isometric to $\bar{C}_{\kappa}^{R}(\Sigma) / x \sim \phi(x)$ and $R \leq \pi /(2 \sqrt{\kappa})$ or $R=\pi / \sqrt{\kappa}$ for $\kappa>0$, where $\phi: \Sigma \times\{R\} \rightarrow \Sigma \times\{R\}$ is an isometric involution (which can be trivial). Conversely, given any isometric involution $\phi$ on $\Sigma$, $\bar{C}_{\kappa}^{R}(\Sigma) / x \sim \phi(x) \in \mathscr{A}_{\kappa}^{R}(\Sigma)$ and has the relatively maximum volume.

Theorem B verifies Conjecture 0.1 for the case $f=g \exp _{p}: Z=\bar{C}_{\kappa}^{R}\left(\Sigma_{p}\right) \rightarrow X$, together with a further classification for the boundary identification. Note that Theorem B implies that if $k>0$ and $\pi /(2 \sqrt{\kappa})<R<\pi / \sqrt{\kappa}$,

$$
\max \left\{\operatorname{vol} X, X \in \mathscr{A}_{\kappa}^{R}(\Sigma)\right\}<v(\Sigma, \kappa, R) .
$$

For the case where $X$ is a limit of Riemannian manifolds, a classification was given in [Grove and Petersen 1992]. A general classification is more complicated, and we wish to discuss it elsewhere. 
As mentioned earlier, Theorem B extends this radius-volume rigidity theorem: Theorem 0.3 [Grove and Petersen 1992]. Let $M_{i} \stackrel{d_{G H}}{\longrightarrow} X$ be a Gromov-Hausdorff convergent sequence of Riemannian n-manifolds such that

$$
\sec _{M_{i}} \geq \kappa, \quad \operatorname{rad}\left(M_{i}\right)=R, \quad \operatorname{vol} M_{i} \rightarrow \operatorname{vol} C_{\kappa}^{R}\left(S_{1}^{n-1}\right),
$$

where $\operatorname{rad}\left(M_{i}\right)=\min \left\{r, \bar{B}_{r}(p)=M_{i}, p \in M_{i}\right\}$. Then $R \leq \pi /(2 \sqrt{\kappa})$ or $R=\pi / \sqrt{\kappa}$ for $\kappa>0$, and $X$ is isometric to the quotient of $\bar{C}_{\kappa}^{R}\left(S_{1}^{n-1}\right)$ by the equivalence relation $x \sim \phi(x)$, where $\phi: \partial \bar{C}_{\kappa}^{R}\left(S_{1}^{n-1}\right) \rightarrow \partial \bar{C}_{\kappa}^{R}\left(S_{1}^{n-1}\right)$ is either the antipodal map or a reflection in a totally geodesic hypersurface. Moreover, each $M_{i}$ is homeomorphic to an $n$-sphere or a real projective $n$-space.

Note that vol $X=\operatorname{vol} C_{\kappa}^{R}\left(S_{1}^{n-1}\right)$. Choosing $p_{i} \in M_{i}$ such that $M_{i}=\bar{B}_{R}\left(p_{i}\right)$, $p_{i} \rightarrow p \in X$ and $\Sigma_{p}=S_{1}^{n-1}$. By now Theorem B implies the rigidity part of Theorem 0.3 (a generalization of the homeomorphic rigidity in Theorem 0.3 will be given in Theorem $\mathrm{C}$ ). Theorem $\mathrm{B}$ also implies the following extension of Theorem 0.3.

Theorem 0.4 [Shteingold 1994]. Let $X \in \mathscr{A}_{\kappa}^{r}\left(S_{1}^{n-1}\right)$ with $\operatorname{vol} X=v\left(S_{1}^{n-1}, \kappa, r\right)$. Then $X=\bar{C}_{\kappa}^{r}\left(S_{1}^{n-1}\right) / x \sim \phi(x), x \in S_{1}^{n-1} \times\{r\}$, where $\phi$ is the reflection on an $\ell$-dimensional totally geodesic subsphere, $1 \leq \ell \leq n(\phi$ is trivial for $\ell=n$.)

A further problem concerning Theorem B is to determine the homeomorphic type of $X$. We have solved this problem for $X$ being a topological manifold (see Theorem 0.3).

Theorem C. Given $\Sigma \in \operatorname{Alex}^{n-1}(1), \kappa$ and $R>0$, there exists a constant $\epsilon=$ $\epsilon(\Sigma, \kappa, R)>0$ such that if $X \in \mathscr{A}_{\kappa}^{R}(\Sigma)$ with $\operatorname{vol} X>v(\Sigma, \kappa, R)-\epsilon$ and $X$ is a closed topological manifold, $X$ is homeomorphic to $S_{1}^{n}$ or a real projective space $\mathbb{R} P^{n}$.

Note that $\Sigma$ in Theorem $C$ is not necessarily a topological manifold; for instance, $X=C_{1}\left(C_{1}(N)\right)$, the twice spherical suspensions over a Poincaré sphere $N$, satisfies Theorem $\mathrm{C}$, but $\Sigma=C_{1}(N)$ is not a topological manifold. However, $X$ is homeomorphic to a 5 -sphere, by [Kapovitch 2002].

In the proof of Theorem B, we establish a pointed version of the Bishop volume comparison with rigidity (Theorem 2.1). In general, we will prove the following pointed version of the Bishop-Gromov relative volume comparison with rigidity.

For $p \in X \in \operatorname{Alex}^{n}(\kappa)$, let $A_{R}^{r}(p)$ denote the annulus $\{x \in X: r<|p x|<R\}$, $0 \leq r<R$, and let $A_{R}^{r}\left(\Sigma_{p}\right)$ denote the corresponding annulus in $C_{\kappa}\left(\Sigma_{p}\right)$.

Theorem D (pointed Bishop-Gromov relative volume comparison). Let

$$
X \in \operatorname{Alex}^{n}(\kappa) .
$$


Then, for any $p \in X$ and $R_{3}>R_{2}>R_{1} \geq 0$,

$\frac{\operatorname{vol} A_{R_{3}}^{R_{1}}(p)}{\operatorname{vol} A_{R_{3}}^{R_{2}}(p)} \geq \frac{\operatorname{vol} A_{R_{3}}^{R_{1}}\left(\Sigma_{p}\right)}{\operatorname{vol} A_{R_{3}}^{R_{2}}\left(\Sigma_{p}\right)}, \quad$ or equivalently, $\quad \frac{\operatorname{vol} A_{R_{2}}^{R_{1}}(p)}{\operatorname{vol} A_{R_{3}}^{R_{2}}(p)} \geq \frac{\operatorname{vol} A_{R_{2}}^{R_{1}}\left(\Sigma_{p}\right)}{\operatorname{vol} A_{R_{3}}^{R_{2}}\left(\Sigma_{p}\right)}$.

In particular,

$$
\frac{\operatorname{vol} B_{R_{1}}(p)}{\operatorname{vol} B_{R_{3}}(p)} \geq \frac{\operatorname{vol} C_{\kappa}^{R_{1}}\left(\Sigma_{p}\right)}{\operatorname{vol} C_{\kappa}^{R_{3}}\left(\Sigma_{p}\right)}
$$

If any of these inequalities becomes an equality, the open ball $B_{R_{3}}(p)$ is isometric to $C_{\kappa}^{R_{3}}\left(\Sigma_{p}\right)$ with respect to the intrinsic metrics.

Remark 0.5. The Riemannian version of the Bishop-Gromov relative comparison for Alexandrov spaces (that is, the model space is $S_{\kappa}^{n}$ ) was stated in [Burago et al. 1992]; compare [Burago et al. 2001]. A notable difference between Theorem D and the Riemannian version is in the rigidity part: the latter is the absolute maximum volume rigidity and its model space is unique, while the former may be viewed as the relatively maximum volume rigidity (relatively to $\Sigma_{p}$ ), whose model spaces are of infinitely many possibilities. Moreover, the proof of Theorem D is considerably difficult; for instance, a dimension-inductive argument (which works in the Riemannian version) does not work.

Remark 0.6. By Lemma 2.1 in [Li 2010], we see that

$$
\frac{\operatorname{vol} C_{\kappa}^{R}\left(\Sigma_{p}\right)}{\operatorname{vol} C_{\kappa}^{r}\left(\Sigma_{p}\right)}=\frac{\operatorname{vol} B_{R}\left(S_{\kappa}^{n}\right)}{\operatorname{vol} B_{r}\left(S_{\kappa}^{n}\right)}
$$

and thus the monotonicity part of Theorem D coincides with that in the Riemannian version. We point out that our proof of the volume ratio monotonicity in Theorem $\mathrm{D}$ is different from one suggested in [Burago et al. 1992]; we take an elementary (calculus) approach via finding an (unconventional) partition suitable for triangle comparison arguments, while a proof in [Burago et al. 2001] relies on a coarea formula for Alexandrov spaces. We point out that in the case where $\kappa \leq 0$, a weak form of the above monotonicity was previously obtained in [Liu and Shen 1994, Proposition 1].

We now give some indication on our approach to Theorem A and Theorem B. In the proof of Theorem A, we show that $f$ is a homeomorphism and $f$ preserves the length of curves. Based on basic properties of an Alexandrov space (not necessarily complete), any curve $c$ in $X$ can be approximated by piecewise geodesics $c_{i}$ in $X^{\delta_{i}}$ $\left(\delta_{i} \rightarrow 0\right)$ such that lengths $L\left(c_{i}\right) \rightarrow L(c)$. Thus, it suffices to show that when restricting to $f^{-1}\left(X^{\delta}\right)$ and $X^{\delta}$, respectively, $f$ is injective and $f^{-1}$ preserves the length of any geodesic up to an error $\tau(\delta) \rightarrow 0$ as $\delta \rightarrow 0$, respectively. We derive this with a volume formula for tube-like $\epsilon$-balls in $X^{\delta}$, which can be treated as a 
replacement of the volume formula of a thin tube around a curve. The proof of the volume formula is based on the fact that a small ball at an $(n, \delta)$-strained point can be almost isometrically embedded into $\mathbb{R}^{n}$; see [Burago et al. 1992].

Our approach to Theorem B consists of two steps: first, establishing the open ball rigidity: the gradient-exponential map $g \exp _{p}: C_{\kappa}^{R}\left(\Sigma_{p}\right) \rightarrow B_{R}(p) \subset X$ is an isometry with respect to the intrinsic distance. We achieve this by showing that $g \exp _{p}$ satisfies the condition in Theorem A; see Lemmas 2.4 and 2.5. Consequently, $X=\bar{C}_{\kappa}^{R}\left(\Sigma_{p}\right) / \sim$, where $\sim$ is a relation on $\Sigma_{p} \times\{R\}: \tilde{x} \sim \tilde{y}$ if and only if $g \exp _{p}(\tilde{x})=g \exp _{p}(\tilde{y})$. Observe that if $\tilde{x} \neq \tilde{y} \in \Sigma_{p} \times\{R\}$ with $\tilde{x} \sim \tilde{y}$, then the $g \exp _{p}$-images of the two geodesics $[\tilde{o} \tilde{x}]$ and $[\tilde{o} \tilde{y}]$ together form a local geodesic at $g \exp _{p} \tilde{x}=g \exp _{p} \tilde{y}$. Because a geodesic does not bifurcate, any equivalent class contains at most two points and thus we obtain an involution $\phi: \Sigma_{p} \times\{R\} \rightarrow \Sigma_{p} \times\{R\}$ such that $X=\bar{C}_{\kappa}^{R}(\Sigma) / \tilde{x} \sim \phi(\tilde{x}), \tilde{x} \in \Sigma_{p} \times\{R\}$. The main difficulty is to show that $\phi$ is an isometry. Our main technical lemma says that $\phi$ is almost 1-bi-Lipschitz up to a uniform error:

$$
\left|\frac{|\phi(\tilde{x}) \phi(\tilde{y})|}{|\tilde{x} \tilde{y}|}-1\right| \leq 20 \tilde{x} \tilde{y} \mid
$$

for $|\tilde{x} \tilde{y}|$ small (see Lemma 2.12). This implies that $\phi$ is continuous and preserves the length of a path, and thus $\phi$ is distance-nonincreasing. Consequently, $\phi$ is an isometry since $\phi$ is an involution. Note that without the curvature lower bound, this does not, in general, imply that the metric on $X=\bar{C}_{\kappa}^{R}(\Sigma) / \tilde{x} \sim \phi(\tilde{x})$ coincides with the induced metric. For example, $X=\bar{C}_{0}^{1}\left(\mathbb{S}_{1}^{1}\right) /(\tilde{x} \sim \tilde{x})=\bar{B}_{1}\left(\mathbb{R}^{2}\right)$ is equipped with the length metric and coincides with the Euclidean metric when restricted to the interior, and $L(\gamma)$ is half of the Euclidean arc length for any $\gamma \subset \partial X$. Our proof relies on the curvature lower bound as well as the cone metric.

Let $L_{p}(X)=g \exp _{p}(\Sigma \times\{R\})$, which locally divides a tubular neighborhood of $L_{p}(X)$ into two components $U_{1}, U_{2}$. The main difficulty in proving the above inequality is that a geodesic in $X$ connecting two points $a, b \in L_{p}(X)$ may intersect with $L_{p}(X)$ at many points other than $a, b$ (called crossing points). We show that if a geodesic is not contained in $L_{p}(X)$, the crossing points are discrete (Corollary 2.9). Thus we can reduce the proof to the case where $c_{1}=[a b] \subset U_{1}$ has no crossing point. It's sufficient to construct a noncrossing piecewise intrinsic geodesic $c_{2} \subset U_{2}$ connecting $a, b$, and show that length $\left(c_{2}\right)$ is close to length $\left(c_{1}\right)=|a b|$ up to a second order error (Lemma 2.12).

We remark that the present proof, in an essential way, relies on the $\kappa$-cone metric structure; and we believe that establishing a similar inequality in general will be the main obstacle in Conjecture 0.1.

Theorems A, B, C and D are proved in Sections 1, 2, 3 and 4, respectively. 


\section{Proof of Theorem A: $(n, \delta)$-strained isometries}

Let $f: Z \rightarrow X$ be as in Theorem A. We will establish that $f$ is an isometry through the following properties:

(i) If a distance-nonincreasing onto map $f$ preserves the volume of the total spaces, then $f$ and $f^{-1}$ preserve volumes of any subsets (see Lemma 1.1).

(ii) Based on a local bi-Lipschitz embedding property (see Lemma 1.2), we show that for $\delta$ suitably small, $f$ is injective on $f^{-1}\left(X^{\delta}\right) \subseteq Z^{\tau(\delta)}$. In particular, for any curve $c \subset X^{\delta}, f^{-1}(c) \subseteq Z^{\tau(\delta)}$ is a curve (see Lemma 1.3).

(iii) Our main technical lemma is a volume formula for a tube of $\epsilon$-balls (which can be treated as a replacement for an $\epsilon$-tube around a curve, see Lemma 1.4). Together with (i) and (ii), this formula implies that $f^{-1}$ preserves the length of any geodesic in $X^{\delta}$ up to an error $\tau(\delta)$. Because for any small $\delta<1 /(8 n)$, the set $X^{\delta}$ is dense in $X$ (see Lemma 1.6), we are able to show that $f$ is also distance nondecreasing and thus $f$ is an isometry.

Lemma 1.1. Let $f: Z \rightarrow X$ be a distance-nonincreasing onto map of two metric spaces of equal Hausdorff dimension. If $\mathrm{vol} X=\operatorname{vol} Z$, then, for any subset $A \subseteq Z$ and $B \subseteq X$,

$$
\operatorname{vol} A=\operatorname{vol} f(A), \quad \operatorname{vol} B=\operatorname{vol} f^{-1}(B) .
$$

Proof. We argue by contradiction. If vol $A>\operatorname{vol} f(A)$, then

$$
\operatorname{vol} Z=\operatorname{vol} A+\operatorname{vol}(Z-A)>\operatorname{vol} f(A)+\operatorname{vol} f(Z-A) \geq \operatorname{vol} f(Z)=\operatorname{vol} X,
$$

a contradiction. Similarly, one can check that vol $f^{-1}(B)=\operatorname{vol} B$.

Let $X^{\delta}(\rho)$ denote the union of points with an $(n, \delta)$-strainer $\left\{\left(a_{i}, b_{i}\right)\right\}$ of radius $\rho>0$, where $\rho=\min _{1 \leq i \leq n}\left\{\left|p a_{i}\right|,\left|p b_{i}\right|\right\}>0$.

Lemma 1.2 [Burago et al. 1992, Theorem 9.4]. Let $X \in \operatorname{Alex}^{n}(\kappa)$. If $p \in X^{\delta}(\rho)$, the map $\psi: X \rightarrow \mathbb{R}^{n}$ defined by $\psi(x)=\left(\left|a_{1} x\right|, \ldots,\left|a_{n} x\right|\right)$ maps a small neighborhood $U$ of $p \tau\left(\delta, \delta_{1}\right)$-almost isometrically onto a domain in $\mathbb{R}^{n}$, that is,

$$
|| \psi(x) \psi(y)|-| x y||<\tau\left(\delta, \delta_{1}\right)|x y|
$$

for any $x, y \in U$, where $\delta_{1}=\rho^{-1} \operatorname{diam}(U)$. In particular, $\psi$ is a $\tau(\delta)$-almost isometric embedding when restricting to $B_{\delta \rho}(p)$.

A consequence of Lemma 1.2 is that

$$
1-\tau(\delta) \leq \frac{\operatorname{vol} B_{\epsilon}(p)}{\operatorname{vol} B_{\epsilon}\left(\mathbb{R}^{n}\right)} \leq 1+\tau(\delta)
$$

for any $p \in X^{\delta}(\rho)$ and $\epsilon \leq \delta \rho$. 
Lemma 1.3. Let the assumptions be as in Theorem A. Then $f: f^{-1}\left(X^{\delta}\right) \rightarrow X^{\delta}$ is injective. Consequently, if $\gamma \subset X^{\delta}$ is a continuous curve, $f^{-1}(\gamma)$ is also a continuous curve.

Proof. We argue by contradiction, assuming $z_{1} \neq z_{2} \in f^{-1}\left(X^{\delta}\right)$ such that $f\left(z_{1}\right)=$ $f\left(z_{2}\right)=x$. We may assume that $z_{1}$ and $z_{2}$ have $\tau(\delta)$-strainers of radius $\rho>0$. Choose $4 \epsilon<\left|z_{1} z_{2}\right|$ and $\epsilon<\delta \rho$. By Lemma 1.1 and the above consequence of Lemma 1.2, we get

$$
1=\frac{\operatorname{vol} f^{-1}\left(B_{\epsilon}(x)\right)}{\operatorname{vol} B_{\epsilon}(x)} \geq \frac{\operatorname{vol} B_{\epsilon}\left(z_{1}\right)+\operatorname{vol} B_{\epsilon}\left(z_{2}\right)}{\operatorname{vol} B_{\epsilon}(x)} \geq 2(1-\tau(\delta)),
$$

a contradiction.

We now develop a formula which estimates the volume of an $\epsilon$-ball tube with a higher order error. Let $x_{1}, x_{2}, \ldots, x_{N+1}$ be $N+1$ points in $X^{\delta}(\rho)$. We first give an estimate of the volume of the $\epsilon$-ball tube $\bigcup_{i=1}^{N+1} B_{\epsilon}\left(x_{i}\right)$ in terms of $\sum_{i=1}^{N}\left|x_{i} x_{i+1}\right|$ and $\epsilon, \delta$ with errors.

Lemma 1.4 (volume of an $\epsilon$-ball tube). Let $X \in \operatorname{Alex}^{n}(\kappa)$ and $x_{i} \in X^{\delta}(\rho), i=$ $1,2, \ldots, N+1$ satisfy that $0<\left|x_{i} x_{i+1}\right|<2 \epsilon \ll \delta \rho$ and $B_{\epsilon}\left(x_{i}\right) \cap B_{\epsilon}\left(x_{j}\right) \cap B_{\epsilon}\left(x_{k}\right)=\varnothing$ for $i \neq j \neq k$. Then the volume of the $\epsilon$-ball tube $\bigcup_{i=1}^{N+1} B_{\epsilon}\left(x_{i}\right)$ (see Figure 1) satisfies

$$
\begin{aligned}
(1+\tau(\delta)) \operatorname{vol} \bigcup_{i=1}^{N+1} B_{\epsilon}\left(x_{i}\right)=\operatorname{vol} B_{\epsilon} & \left(\mathbb{R}^{n}\right) \\
& +2 \epsilon \operatorname{vol} B_{\epsilon}\left(\mathbb{R}^{n-1}\right) \sum_{i=1}^{N} \int_{\theta_{i}}^{\pi / 2} \sin ^{n}(t) d t,
\end{aligned}
$$

where $\theta_{i} \in[0, \pi / 2]$ such that $\cos \theta_{i}=\left|x_{i} x_{i+1}\right| /(2 \epsilon)$. If, in addition, $\left|x_{i} x_{i+1}\right| \leq \epsilon^{2}$ for all $1 \leq i \leq N$,

$$
\begin{aligned}
(1+\tau(\delta)) \operatorname{vol} \bigcup_{i=1}^{N+1} B_{\epsilon}( & \left.x_{i}\right)=\operatorname{vol} B_{\epsilon}\left(\mathbb{R}^{n}\right) \\
& +\operatorname{vol} B_{\epsilon}\left(\mathbb{R}^{n-1}\right) \sum_{i=1}^{N}\left|x_{i} x_{i+1}\right|+O\left(\epsilon^{n+1}\right) \sum_{i=1}^{N}\left|x_{i} x_{i+1}\right| .
\end{aligned}
$$

Because $B_{\epsilon}\left(x_{i-1}\right) \cup B_{\epsilon}\left(x_{i}\right) \cup B_{\epsilon}\left(x_{i+1}\right) \subset B_{\delta \rho}\left(x_{i}\right)$, which is $\tau(\delta)$-almost isometrically embedded into $\mathbb{R}^{n}$, one can divide $\bigcup_{i=1}^{N+1} B_{\epsilon}\left(x_{i}\right)$ into small pieces $\Gamma^{ \pm}\left(x_{i}\right)$, whose volumes are $(1+\tau(\delta))$-proportional to the volumes of the following "trapezoidal balls"

$$
\Gamma_{\epsilon}^{h_{i}^{ \pm}}\left(\mathbb{R}^{n}\right)
$$

in $\mathbb{R}^{n}$. This allows us to reduce the calculation to Euclidean space.

We define the trapezoidal ball $\Gamma_{r}^{h}\left(\mathbb{R}^{n}\right)$ in $\mathbb{R}_{+}^{n}=\left\{\left(x_{1}, x_{2}, \ldots, x_{n}\right): x_{n} \geq 0\right\}$ in the following way. Let $u \in \mathbb{R}_{+}^{n}$ be a point with $|o u|=h \leq r$. Then the hyper plane $H$ 


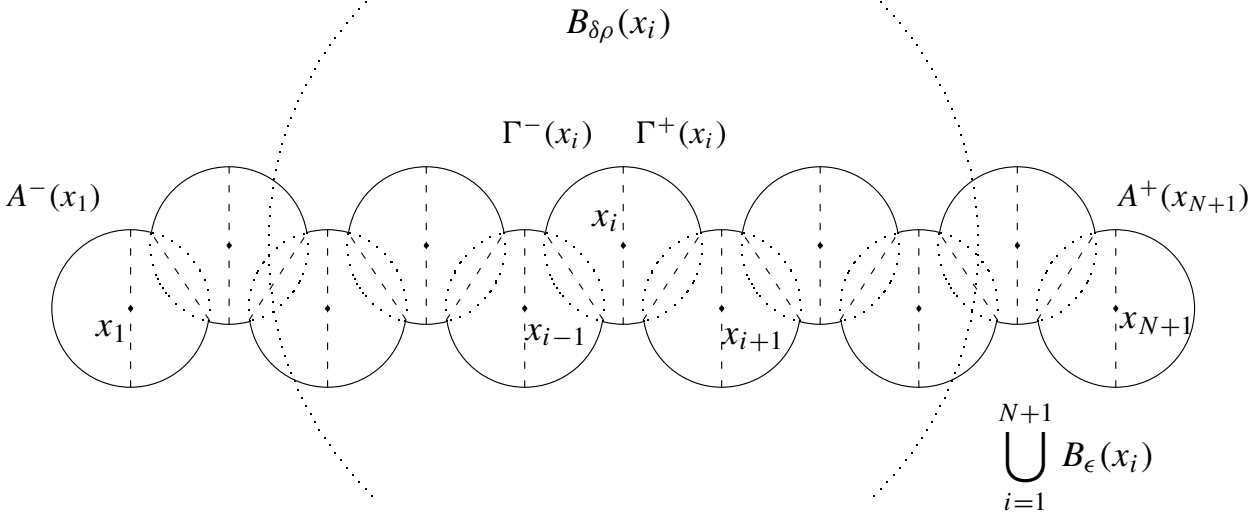

Figure 1

passing through $u$ and perpendicular to $\overrightarrow{o u}$ divides the half ball $B_{r}\left(\mathbb{R}^{n}\right) \cap \mathbb{R}_{+}^{n}$ into two subsets. Let $\Gamma_{r}^{h}\left(\mathbb{R}^{n}\right)$ be the subset which contains the origin (see Figure 3 ). It's easy to see that vol $\Gamma_{r}^{h}\left(\mathbb{R}^{n}\right)$ depends only on $h$ and $r$, and not on the direction $\overrightarrow{o u}$, as long as $H \cap B_{r}\left(\mathbb{R}^{n}\right) \subset \mathbb{R}_{+}^{n}$.

Lemma 1.5. Let $\Gamma_{r}^{h}\left(\mathbb{R}^{n}\right)$ be a trapezoidal ball defined as above. Then

$$
\operatorname{vol} \Gamma_{r}^{h}\left(\mathbb{R}^{n}\right)=r \operatorname{vol} B_{r}\left(\mathbb{R}^{n-1}\right) \int_{\theta}^{\pi / 2} \sin ^{n}(t) d t,
$$

where $\theta \in[0, \pi / 2]$ such that $r \cos \theta=h$.

Proof. Let $s=r \cos t \in[0, h]$ be the parameter for the height with the corresponding angle $t \in[\theta, \pi / 2]$. Then

$$
\begin{aligned}
\operatorname{vol} \Gamma_{r}^{h}\left(\mathbb{R}^{n}\right) & =\int_{0}^{h} \operatorname{vol} B_{r \sin t}\left(\mathbb{R}^{n-1}\right) d s=\int_{\theta}^{\pi / 2} \operatorname{vol} B_{r \sin t}\left(\mathbb{R}^{n-1}\right) r \sin (t) d t \\
& =r \operatorname{vol} B_{r}\left(\mathbb{R}^{n-1}\right) \int_{\theta}^{\pi / 2} \sin ^{n}(t) d t .
\end{aligned}
$$

Proof of the volume formula, Lemma 1.4. Because $B_{\epsilon}\left(x_{i}\right) \cap B_{\epsilon}\left(x_{i+1}\right) \neq \varnothing$ and $B_{\epsilon}\left(x_{i}\right) \cap B_{\epsilon}\left(x_{j}\right) \cap B_{\epsilon}\left(x_{k}\right)=\varnothing$ for any $i \neq j \neq k$, we can decompose $\bigcup_{i=1}^{N+1} B_{\epsilon}\left(x_{i}\right)$ as the following (see Figure 2): let

$$
A^{+}\left(x_{i}\right)=\left\{q \in B_{\epsilon}\left(x_{i}\right):\left|q x_{i}\right| \leq\left|q x_{i+1}\right|\right\}, \quad A^{-}\left(x_{i}\right)=\left\{q \in B_{\epsilon}\left(x_{i}\right):\left|q x_{i}\right| \leq\left|q x_{i-1}\right|\right\} .
$$

For $i=2,3, \ldots, N$, let

$$
\begin{aligned}
& H^{+}\left(x_{i}\right)=A^{+}\left(x_{i}\right) \cap A^{-}\left(x_{i+1}\right)=\left\{q \in B_{\epsilon}\left(x_{i}\right) \cap B_{\epsilon}\left(x_{i+1}\right):\left|q x_{i}\right|=\left|q x_{i+1}\right|\right\}, \\
& H^{-}\left(x_{i}\right)=A^{-}\left(x_{i}\right) \cap A^{+}\left(x_{i-1}\right)=\left\{q \in B_{\epsilon}\left(x_{i}\right) \cap B_{\epsilon}\left(x_{i-1}\right):\left|q x_{i}\right|=\left|q x_{i-1}\right|\right\},
\end{aligned}
$$



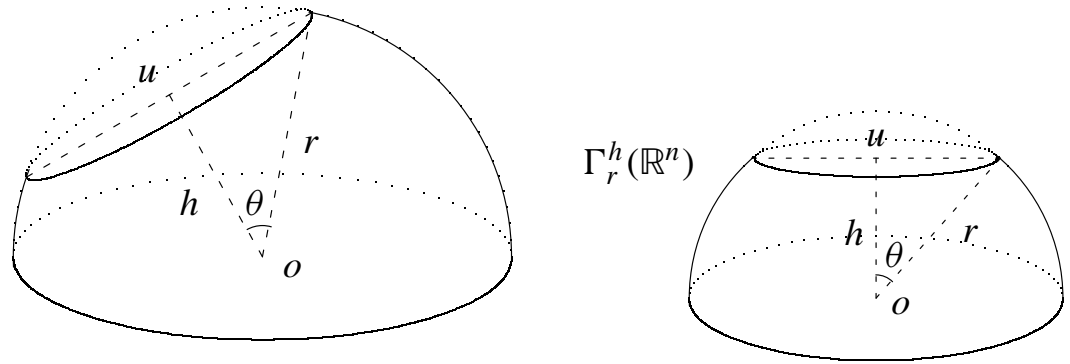

Figure 2

and

$$
\begin{aligned}
& \Gamma^{+}\left(x_{i}\right)=\left\{q \in A^{+}\left(x_{i}\right) \cap A^{-}\left(x_{i}\right): d\left(q, H^{+}\left(x_{i}\right)\right) \leq d\left(q, H^{-}\left(x_{i}\right)\right)\right\}, \\
& \Gamma^{-}\left(x_{i}\right)=\left\{q \in A^{+}\left(x_{i}\right) \cap A^{-}\left(x_{i}\right): d\left(q, H^{+}\left(x_{i}\right)\right) \geq d\left(q, H^{-}\left(x_{i}\right)\right)\right\} .
\end{aligned}
$$

By the construction,

$$
\bigcup_{i=1}^{N+1} B_{\epsilon}\left(x_{i}\right)=A^{-}\left(x_{1}\right) \cup\left(\bigcup_{i=2}^{N} \Gamma^{ \pm}\left(x_{i}\right)\right) \cup A^{+}\left(x_{N+1}\right) .
$$

Note that $H^{ \pm}\left(x_{i}\right), i=2, \ldots, N$ consist of all the possible intersections of any two of $A^{-}\left(x_{1}\right), \Gamma^{ \pm}\left(x_{i}\right), i=2, \ldots, N$, and $A^{+}\left(x_{N+1}\right)$ and vol $H^{ \pm}\left(x_{i}\right)=0$. We have

$$
\begin{aligned}
& \operatorname{vol} \bigcup_{i=1}^{N+1} B_{\epsilon}\left(x_{i}\right) \\
& \quad=\operatorname{vol} A^{-}\left(x_{1}\right)+\operatorname{vol} A^{+}\left(x_{N+1}\right)+\sum_{i=2}^{N} \operatorname{vol} \Gamma^{+}\left(x_{i}\right)+\sum_{i=2}^{N} \operatorname{vol} \Gamma^{-}\left(x_{i}\right) .
\end{aligned}
$$

Because $B_{\epsilon}\left(x_{i-1}\right) \cup B_{\epsilon}\left(x_{i}\right) \cup B_{\epsilon}\left(x_{i+1}\right) \subset B_{\delta \rho}\left(x_{i}\right)$, which is homeomorphically and $\tau(\delta)$-almost isometrically embedded into $\mathbb{R}^{n}$, we have that

$$
\begin{aligned}
(1+\tau(\delta)) \operatorname{vol} \Gamma^{ \pm}\left(x_{i}\right) & =\operatorname{vol} \Gamma_{\epsilon}^{h_{i}^{ \pm}}\left(\mathbb{R}^{n}\right), \\
(1+\tau(\delta)) \operatorname{vol} A^{+}\left(x_{1}\right) & =\frac{1}{2} \operatorname{vol} B_{\epsilon}\left(\mathbb{R}^{n}\right)+\operatorname{vol} \Gamma_{\epsilon}^{h_{1}^{+}}\left(\mathbb{R}^{n}\right), \\
(1+\tau(\delta)) \operatorname{vol} A^{-}\left(x_{N+1}\right) & =\frac{1}{2} \operatorname{vol} B_{\epsilon}\left(\mathbb{R}^{n}\right)+\operatorname{vol} \Gamma_{\epsilon}^{h_{N+1}^{-}}\left(\mathbb{R}^{n}\right),
\end{aligned}
$$

where $h_{i}^{+}=\frac{1}{2}\left|x_{i} x_{i+1}\right|, h_{i}^{-}=\frac{1}{2}\left|x_{i} x_{i-1}\right|$. Note that it's our convention that the same symbol $\tau(\delta)$ may represent different functions of $\delta$, as long as $\tau(\delta) \rightarrow 0$ as $\delta \rightarrow 0$. Together with (1-3) and the fact that $h_{i}^{+}=h_{i+1}^{-}$, we get

$$
(1+\tau(\delta)) \operatorname{vol} \bigcup_{i=1}^{N+1} B_{\epsilon}\left(x_{i}\right)=\operatorname{vol} B_{\epsilon}\left(\mathbb{R}^{n}\right)+2 \sum_{i=1}^{N} \operatorname{vol} \Gamma_{\epsilon}^{h_{i}^{+}}\left(\mathbb{R}^{n}\right) .
$$


Let $\theta_{i} \in[0, \pi / 2]$ such that $\cos \theta_{i}=h_{i}^{+} / \epsilon=\left|x_{i} x_{i+1}\right| /(2 \epsilon)$. By Lemma 1.5, we have

$$
\operatorname{vol} \Gamma_{\epsilon}^{h_{i}^{+}}\left(\mathbb{R}^{n}\right)=\epsilon \operatorname{vol} B_{\epsilon}\left(\mathbb{R}^{n-1}\right) \int_{\theta_{i}}^{\pi / 2} \sin ^{n}(t) d t .
$$

Plugging this into (1-4), we get (1-1).

To get (1-2), we need to write $\int_{\theta_{i}}^{\pi / 2} \sin ^{n}(t) d t$ in terms of $\left|x_{i} x_{i+1}\right|$. Let

$$
g(s)=\int_{\theta}^{\pi / 2} \sin ^{n}(t) d t
$$

where $\theta \in[0, \pi / 2]$ with $\cos \theta=s /(2 \epsilon)$. Noting that $\theta=\pi / 2$ if and only if $s=0$, we have $g(0)=0$. Furthermore,

$$
\begin{aligned}
& g^{\prime}(s)=-\sin ^{n} \theta \cdot \frac{d \theta}{d s}=-\sin ^{n} \theta \cdot \frac{1}{-2 \epsilon \sin \theta}=\frac{\sin ^{n-1} \theta}{2 \epsilon} ; \\
& g^{\prime \prime}(s)=\frac{1}{2 \epsilon}(n-1) \sin ^{n-2} \theta \cos \theta \cdot \frac{1}{-2 \epsilon \sin \theta}=\frac{n-1}{-4 \epsilon^{2}} \sin ^{n-3} \theta \cos \theta ;
\end{aligned}
$$

and thus $g^{\prime}(0)=1 /(2 \epsilon), g^{\prime \prime}(0)=0$, and $g^{\prime \prime \prime}(0)=c_{n} / \epsilon^{3}$. The Taylor expansion of $g$ at $s=0$ is

$$
g(s)=\int_{\theta}^{\pi / 2} \sin ^{n}(t) d t=0+\frac{s}{2 \epsilon}+\frac{1}{\epsilon^{3}} \cdot O\left(s^{3}\right) .
$$

Letting $s=\left|x_{i} x_{i+1}\right| \leq \epsilon^{2}$, we get

$$
\int_{\theta_{i}}^{\pi / 2} \sin ^{n}(t) d t=\frac{1}{2 \epsilon}\left|x_{i} x_{i+1}\right|+O(\epsilon)\left|x_{i} x_{i+1}\right| .
$$

Plugging this into (1-1), we get (1-2).

In the rest of this section we assume that $f: Z \rightarrow X$ is a distance-nonincreasing onto map such that $f^{-1}\left(X^{\delta}\right) \subset Z^{\tau(\delta)}$. By Lemma 1.3, $f$ is homeomorphic on $f^{-1}\left(X^{\delta}\right)$.

Lemma 1.6. Let the assumptions be as in Theorem A. Let $x, y \in X^{\delta}$. For $\delta>0$ sufficiently small, there exists a small constant $c=c(\rho, \delta)>0$ such that if $|x y| \leq c$, $\left|f^{-1}(x) f^{-1}(y)\right| \leq 2|x y|$.

Proof. Assume that $|x y|=\epsilon \ll \delta \rho$ and $\left|f^{-1}(x) f^{-1}(y)\right|>2 \epsilon$. Consider the metric balls $B_{\epsilon}(x)$ and $B_{\epsilon}(y)$. By Lemma 1.4,

$$
\begin{aligned}
(1+\tau(\delta)) \operatorname{vol}\left(B_{\epsilon}(x)\right. & \left.\cup B_{\epsilon}(y)\right) \\
& =\operatorname{vol} B_{\epsilon}\left(\mathbb{R}^{n}\right)+2 \epsilon \operatorname{vol} B_{\epsilon}\left(\mathbb{R}^{n-1}\right) \int_{\pi / 3}^{\pi / 2} \sin ^{n}(t) d t+O\left(\epsilon^{n+1}\right) .
\end{aligned}
$$

Since $B_{\epsilon}\left(f^{-1}(x)\right) \cap B_{\epsilon}\left(f^{-1}(y)\right)=\varnothing$, we have

$$
(1+\tau(\delta)) \operatorname{vol}\left(B_{\epsilon}\left(f^{-1}(x)\right) \cup B_{\epsilon}\left(f^{-1}(y)\right)\right)=2 \operatorname{vol} B_{\epsilon}\left(\mathbb{R}^{n}\right) .
$$


Because $f$ is distance-nonincreasing,

$$
B_{\epsilon}\left(f^{-1}(x)\right) \cup B_{\epsilon}\left(f^{-1}(y)\right) \subset f^{-1}\left(B_{\epsilon}(x) \cup B_{\epsilon}(y)\right) .
$$

Together with the fact that $f^{-1}$ is volume-preserving, we get

$$
\begin{aligned}
1 & =\frac{\operatorname{vol} f^{-1}\left(B_{\epsilon}(x) \cup B_{\epsilon}(y)\right)}{\operatorname{vol}\left(B_{\epsilon}(x) \cup B_{\epsilon}(y)\right)} \\
& \geq \frac{(1-\tau(\delta)) \cdot 2 \operatorname{vol} B_{\epsilon}\left(\mathbb{R}^{n}\right)}{\operatorname{vol} B_{\epsilon}\left(\mathbb{R}^{n}\right)+2 \epsilon \operatorname{vol} B_{\epsilon}\left(\mathbb{R}^{n-1}\right) \int_{\pi / 3}^{\pi / 2} \sin ^{n}(t) d t+O\left(\epsilon^{n+1}\right)} \\
& =\frac{(1-\tau(\delta)) \cdot 2 \int_{0}^{\pi / 2} \sin ^{n}(t) d t}{\int_{0}^{\pi / 2} \sin ^{n}(t) d t+\int_{\pi / 3}^{\pi / 2} \sin ^{n}(t) d t+O(\epsilon)} .
\end{aligned}
$$

(See Lemma 1.5, $\theta=0$.) This leads to a contradiction for sufficiently small $\epsilon$ and $\delta$.

In the proof of Theorem A, we will need the following result.

Lemma 1.7 [Burago et al. 1992, 10.6.1]. Let $X \in \operatorname{Alex}^{n}(\kappa)$. For a fixed sufficiently small $\delta>0$, the union of interior points which do not admit any $(n, \delta)$-strainer has Hausdorff dimension $\leq n-2$. In particular, $X^{\delta}$ is dense.

Proof of Theorem A. Since $f$ is distance-nonincreasing, it suffices to show that $f$ is distance nondecreasing, that is, for any $\tilde{a}, \tilde{b} \in Z,|a b| \geq|\tilde{a} \tilde{b}|$, where $a=f(\tilde{a})$ and $b=f(\tilde{b})$.

For any small $\epsilon_{1}$, by Lemma 1.7, there are $\tilde{a}_{\epsilon_{1}}, \tilde{b}_{\epsilon_{1}} \in Z^{\tau(\delta)}, a_{\epsilon_{1}}=f\left(\tilde{a}_{\epsilon_{1}}\right), b_{\epsilon_{1}}=$ $f\left(\tilde{b}_{\epsilon_{1}}\right) \in X^{\delta}$, such that $\left|a a_{\epsilon_{1}}\right| \leq\left|\tilde{a} \tilde{a}_{\epsilon_{1}}\right|<\epsilon_{1},\left|b b_{\epsilon_{1}}\right| \leq\left|\tilde{b} \tilde{b}_{\epsilon_{1}}\right|<\epsilon_{1}$.

Case 1. Assume that there exists a minimal geodesic $\left[a_{\epsilon_{1}} b_{\epsilon_{1}}\right] \subset X$. Then, because the spaces of directions are isometric along the interior of a geodesic, $\left[a_{\epsilon_{1}} b_{\epsilon_{1}}\right] \subset X^{2 \delta}$ [Petrunin 1998]. By Lemma 1.3 (which will be frequently used without mention), $f^{-1}\left(\left[a_{\epsilon_{1}} b_{\epsilon_{1}}\right]\right)$ is also a continuous curve. Because $\left[a_{\epsilon_{1}} b_{\epsilon_{1}}\right]$ is compact, we may let $\rho>0$ such that $\left[a_{\epsilon_{1}} b_{\epsilon_{1}}\right] \subset X^{2 \delta}(\rho)$ and $f^{-1}\left(\left[a_{\epsilon_{1}} b_{\epsilon_{1}}\right]\right) \subset Z^{\tau(\delta)}(\rho)$. Let $\left\{x_{i}\right\}_{i=1}^{N+1}$ be an $\epsilon$-partition of $\left[a_{\epsilon_{1}} b_{\epsilon_{1}}\right]$, where $x_{1}=a_{\epsilon_{1}}, x_{N+1}=b_{\epsilon_{1}}$ for $\epsilon \ll \delta \rho$. Because $\left[a_{\epsilon_{1}} b_{\epsilon_{1}}\right]$ is a geodesic, Lemma 1.4 can be applied on the partition $\left\{x_{i}\right\}_{i=1}^{N+1}$. Thus we get

$$
\begin{aligned}
(1+\tau(\delta)) \operatorname{vol} \bigcup_{i=1}^{N+1} & B_{\epsilon}\left(x_{i}\right) \\
& =\operatorname{vol} B_{\epsilon}\left(\mathbb{R}^{n}\right)+\operatorname{vol} B_{\epsilon}\left(\mathbb{R}^{n-1}\right) \sum_{i=1}^{N}\left|x_{i} x_{i+1}\right|+O\left(\epsilon^{n+1}\right) \sum_{i=1}^{N}\left|x_{i} x_{i+1}\right| \\
& =\operatorname{vol} B_{\epsilon}\left(\mathbb{R}^{n-1}\right)\left|a_{\epsilon_{1}} b_{\epsilon_{1}}\right|+O\left(\epsilon^{n}\right) .
\end{aligned}
$$

Let $z_{i}=f^{-1}\left(x_{i}\right)$. By Lemma 1.6, $\left|z_{i} z_{i+1}\right| \leq 2\left|x_{i} x_{i+1}\right|=2 \epsilon$. Together with the fact that $f$ is distance-nonincreasing, one can easily check that $\bigcup_{i=1}^{N+1} B_{\epsilon}\left(z_{i}\right)$ satisfies 
the condition of Lemma 1.4. Then we have

$$
(1+\tau(\delta)) \operatorname{vol} \bigcup_{i=1}^{N+1} B_{\epsilon}\left(z_{i}\right)=\operatorname{vol} B_{\epsilon}\left(\mathbb{R}^{n-1}\right) \sum_{i=1}^{N}\left|z_{i} z_{i+1}\right|+O\left(\epsilon^{n}\right) .
$$

Because $f$ is distance-nonincreasing and volume-preserving,

$$
\begin{aligned}
1 & =\frac{\operatorname{vol} f^{-1}\left(\bigcup_{i=1}^{N+1} B_{\epsilon}\left(x_{i}\right)\right)}{\operatorname{vol} \bigcup_{i=1}^{N+1} B_{\epsilon}\left(x_{i}\right)} \geq \frac{\operatorname{vol} \bigcup_{i=1}^{N+1} B_{\epsilon}\left(z_{i}\right)}{\operatorname{vol} \bigcup_{i=1}^{N+1} B_{\epsilon}\left(x_{i}\right)} \\
& =(1-\tau(\delta)) \frac{\operatorname{vol} B_{\epsilon}\left(\mathbb{R}^{n-1}\right) \sum_{i=1}^{N}\left|z_{i} z_{i+1}\right|+O\left(\epsilon^{n}\right)}{\operatorname{vol} B_{\epsilon}\left(\mathbb{R}^{n-1}\right)\left|a_{\epsilon_{1}} b_{\epsilon_{1}}\right|+O\left(\epsilon^{n}\right)}, \\
& =(1-\tau(\delta)) \frac{\sum_{i=1}^{N}\left|z_{i} z_{i+1}\right|+O(\epsilon)}{\left|a_{\epsilon_{1}} b_{\epsilon_{1}}\right|+O(\epsilon)} \\
& \geq(1-\tau(\delta)) \frac{\left|\tilde{a}_{\epsilon_{1}} \tilde{b}_{\epsilon_{1}}\right|+O(\epsilon)}{\left|a_{\epsilon_{1}} b_{\epsilon_{1}}\right|+O(\epsilon)}
\end{aligned}
$$

Letting $\epsilon \rightarrow 0$, we get

$$
\left|a_{\epsilon_{1}} b_{\epsilon_{1}}\right| \geq(1-\tau(\delta))\left|\tilde{a}_{\epsilon_{1}} \tilde{b}_{\epsilon_{1}}\right| .
$$

Case 2. Assume that there is no minimal geodesic in $X^{\delta}$ from $a_{\epsilon_{1}}$ to $b_{\epsilon_{1}}$ (since $X$ may not be complete). Because spaces of directions along the interior of a geodesic are isometric to each other [Petrunin 1997], we may assume a curve $c_{1}$ in $X^{\delta}$ from $a_{\epsilon_{1}}$ to $b_{\epsilon_{1}}$ such that $L\left(c_{1}\right)<\left|a_{\epsilon_{1}} b_{\epsilon_{1}}\right|+\epsilon_{1}$. Since $c_{1}(t)$ is a compact subset in the open set $X^{\delta}$, we may assume $\eta>0$ such that an $\eta$-tube of $c_{1}$ is also contained in $X^{\delta}$. Consequently, we may assume a piecewise geodesic $c$ in $X^{\delta}$ such that $L(c) \leq L\left(c_{1}\right) \leq\left|a_{\epsilon_{1}} b_{\epsilon_{1}}\right|+\epsilon_{1}$. Applying Case 1 to each geodesic segment of $c$, we conclude that

$$
\left|a_{\epsilon_{1}} b_{\epsilon_{1}}\right| \geq L(c)-\epsilon_{1} \geq(1-\tau(\delta))\left|\tilde{a}_{\epsilon_{1}} \tilde{b}_{\epsilon_{1}}\right|-\epsilon_{1} .
$$

In either Case 1 or Case 2, we have

$$
\begin{aligned}
|a b| & \geq\left|a_{\epsilon_{1}} b_{\epsilon_{1}}\right|-2 \epsilon_{1} \geq(1-\tau(\delta))\left|\tilde{a}_{\epsilon_{1}} \tilde{b}_{\epsilon_{1}}\right|-3 \epsilon_{1} \\
& \geq(1-\tau(\delta))\left(|\tilde{a} \tilde{b}|-2 \epsilon_{1}\right)-3 \epsilon_{1} .
\end{aligned}
$$

Letting $\delta \rightarrow 0, \epsilon_{1} \rightarrow 0$, we get $|a b| \geq|\tilde{a} \tilde{b}|$.

\section{Proof of Theorem B: Relatively maximum volume}

Our proof of the classification part in Theorem B is divided into the following two theorems: open ball rigidity (Theorem 2.1) and isometric involution (Theorem 2.2). Recall that $\tilde{o}$ denotes the vertex of the cone $\bar{C}_{\kappa}^{R}\left(\Sigma_{p}\right)$ and thus $g \exp _{p}(\tilde{o})=p$. 
Theorem 2.1. Under the assumptions of Theorem B,

$$
g \exp _{p}: C_{\kappa}^{R}(\Sigma) \rightarrow B_{R}(p)
$$

is an isometry with respect to the intrinsic metrics. In particular, $g \exp _{p}=\exp _{p}$.

By Theorem 2.1, $X=\bar{C}_{\kappa}^{R}\left(\Sigma_{p}\right) / x \sim x^{\prime}$, where the equivalent relation $x \sim x^{\prime}$ if and only if $\exp _{p} x=\exp _{p} x^{\prime}$ and $x, x^{\prime} \in \Sigma_{p} \times\{R\}$.

Theorem 2.2. Let $X=\bar{C}_{\kappa}^{R}\left(\Sigma_{p}\right) / x \sim x^{\prime} \in \operatorname{Alex}^{n}(\kappa)$ be defined as above. Then each equivalent class contains at most two points. Moreover, the induced involution $\phi: \Sigma_{p} \times\{R\} \rightarrow \Sigma_{p} \times\{R\}, \phi(x)=x^{\prime}$ (where $\left.x \sim x^{\prime}\right)$ is an isometry.

Recall that the induced gradient-exponential map $g \exp _{p}: \bar{C}_{\kappa}^{R}(\Sigma) \rightarrow \bar{B}_{R}(p)=X$ is distance-nonincreasing and onto. Indeed, the open ball rigidity is essentially a consequence of Theorem A and the general property that $\exp _{p}^{-1}: X \rightarrow T_{p} X: \exp _{p}^{-1}$ preserves $(n, \delta)$-strained points up to a constant depending on $\delta$ (see Lemma 2.4). In the proof, let's recall the following property from [Burago et al. 1992]:

Lemma 2.3 [Burago et al. 1992, Lemmas 7.5 and 11.2]. Let $p \in X \in \operatorname{Alex}^{n}(\kappa)$. Then, for any $\delta>0$, there is a small neighborhood $U_{p}$ of $p$ such that, for any triangle $\triangle p a b$ with $a, b \in U_{p}$, each angle of $\triangle p a b \subset X$ differs from the comparison angle of $\tilde{\triangle} p a b \subset \mathbb{S}_{\kappa}^{2}$ by less than $\delta$.

Lemma 2.4. Let $q \in X^{\delta}$. Then for any $p \in X, \uparrow_{p}^{q} \in \Sigma_{p}^{\tau(\delta)}$. Consequently,

$$
\exp _{p}^{-1}(q) \in \bar{C}_{\kappa}^{R}\left(\Sigma_{p}\right)^{\tau(\delta)} .
$$

Proof. Since $q \in X^{\delta}$, by Lemma 1.2, we may assume an $(n, 2 \delta)$-strainer $\left\{\left(a_{i}, b_{i}\right)\right\}$ for $q_{1} \in[p q]$ and near $q$, such that $b_{n}=q, a_{n} \in\left[p q_{1}\right]$. Because the spaces of directions are isometric along the interior of a geodesic [Petrunin 1998], there is $q^{\prime} \in[p q] \cap U_{p}$ which has an $(n, \tau(\delta))$-strainer $\left\{\left(a_{i}^{\prime}, b_{i}^{\prime}\right)\right\}$. By the same reason as above, we can assume that $a_{n}^{\prime} \in\left[p q^{\prime}\right]$ and $b_{n}^{\prime} \in\left[q^{\prime} q\right]$.

In addition, we can assume that $\left|q^{\prime} a_{i}^{\prime}\right|,\left|q^{\prime} b_{i}^{\prime}\right|$ are short so that $a_{i}^{\prime}, b_{i}^{\prime} \in U_{p}$ and $\measuredangle a_{i}^{\prime} p q^{\prime}, \measuredangle b_{i}^{\prime} p q^{\prime}<5 \delta$. We claim that

$$
\left\{\left(\uparrow \stackrel{a_{i}^{\prime}}{p}, \uparrow_{p}^{b_{i}^{\prime}}\right)\right\}_{i=1}^{n-1}
$$

forms an $(n-1, \tau(\delta))$-strainer at $\uparrow_{p}^{q} \in \Sigma_{p}$. It's easy to see that

$$
\measuredangle a_{i}^{\prime} p q^{\prime}=\widetilde{\measuredangle} a_{i}^{\prime} p q^{\prime}+\tau(\delta)=\frac{\left|a_{i}^{\prime} q^{\prime}\right|}{\left|p q^{\prime}\right|}+\tau(\delta) .
$$

Thus

$$
\cos \widetilde{\measuredangle} \uparrow_{p}^{a_{i}^{\prime}} \uparrow_{p}^{q^{\prime}} \uparrow_{p}^{x_{j}}=\frac{\left|a_{i}^{\prime} q^{\prime}\right|^{2}+\left|x_{j} q^{\prime}\right|^{2}-\left|a_{i}^{\prime} x_{j}\right|}{2\left|a_{i}^{\prime} q^{\prime}\right|\left|x_{j} q\right|}+\tau(\delta)=\cos \widetilde{\measuredangle} a_{i}^{\prime} q^{\prime} x_{j}+\tau(\delta),
$$

where $i, j=1,2, \ldots, n-1, x_{j}=a_{j}^{\prime}$ or $b_{j}^{\prime}$. 
To conclude the open ball rigidity by applying Theorem A, we need to check that $g \exp _{p}^{-1}\left(X^{\delta}\right) \subseteq \bar{C}_{\kappa}^{R}\left(\Sigma_{p}\right)^{\tau(\delta)}$. We do this by showing that $g \exp _{p}=\exp _{p}$ when $\operatorname{vol} X=v\left(\Sigma_{p}, \kappa, R\right)$.

Lemma 2.5. If $\operatorname{vol} B_{R}(p)=\operatorname{vol} C_{\kappa}^{R}\left(\Sigma_{p}\right)$, the gradient exponential map is actually an exponential map $\exp _{p}: \bar{C}_{\kappa}^{R}\left(\Sigma_{p}\right) \rightarrow \bar{B}_{R}(p)$ which preserves the distance along the radial direction.

Proof. Clearly, the map $\exp _{p}^{-1}: \bar{B}_{R}(p) \rightarrow \bar{C}_{\kappa}^{R}\left(\Sigma_{p}\right)$ (If there is more than one image, we will pick one) is distance nondecreasing. Because

$$
\operatorname{vol} C_{\kappa}^{R}\left(\Sigma_{p}\right)=\operatorname{vol} X \leq \operatorname{vol} \exp _{p}^{-1}(X) \leq \operatorname{vol} C_{\kappa}^{R}\left(\Sigma_{p}\right),
$$

$\exp _{p}^{-1}(X)$ is dense in $C_{\kappa}^{R}\left(\Sigma_{p}\right)$. For any $z \in C_{\kappa}^{R}\left(\Sigma_{p}\right)$, there is a sequence $x_{i} \in X$, such that $\exp _{p}^{-1}\left(x_{i}\right)=z_{i} \rightarrow z$. Let $\exp _{p}: C_{\kappa}^{R}\left(\Sigma_{p}\right) \rightarrow X ; \exp _{p}(z)=\lim _{i \rightarrow \infty} x_{i}$. Such an $\exp _{p}$ is well defined, since if there is another sequence $\exp _{p}^{-1}\left(x_{i}^{\prime}\right)=z_{i}^{\prime} \rightarrow z$,

$$
d\left(\lim _{i \rightarrow \infty} x_{i}, \lim _{i \rightarrow \infty} x_{i}^{\prime}\right)=\lim _{i \rightarrow \infty} d\left(x_{i}, x_{i}^{\prime}\right) \leq \lim _{i \rightarrow \infty} d\left(z_{i}, z_{i}^{\prime}\right)=0 .
$$

It's clear that $\exp _{p}$, defined as an extension of $\exp _{p}^{-1}$, is distance-nonincreasing. Moreover, it preserves the distance along the radial direction.

We now show that any geodesic from $p=\exp _{p}(\tilde{o})$ to $q=\exp _{p}(\tilde{q}) \in B_{R}(p)$ can be extended. Therefore $\exp _{p}$ is a bijection, since geodesics do not bifurcate. Let $[\tilde{o} \tilde{q}]$ be the geodesic in $C_{\kappa}^{R}\left(\Sigma_{p}\right)$ such that $\exp _{p}([\tilde{o} \tilde{q}])=[p q]$, and $\tilde{q}^{\prime} \in C_{\kappa}^{R}\left(\Sigma_{p}\right)$ the extended point of $[\tilde{o} \tilde{q}]$. Then

$$
|p q|+\left|q q^{\prime}\right| \leq|\tilde{o} \tilde{q}|+\left|\tilde{q} \tilde{q}^{\prime}\right|=\left|\tilde{o} \tilde{q}^{\prime}\right|=\left|p q^{\prime}\right|,
$$

which forces $[p q] \cup\left[q q^{\prime}\right]$ to be a geodesic.

Proof of Theorem 2.1. For $X \in \mathscr{A}_{\kappa}^{R}(\Sigma)$ with vol $X=v(\Sigma, \kappa, R)$, by Lemmas 2.4 and 2.5, we see that $\exp _{p}: C_{\kappa}^{R}(\Sigma) \rightarrow B_{R}(p)$ is a distance-nonincreasing onto map that satisfies the assumptions in Theorem A (note that $\exp _{p}: \bar{C}_{\kappa}^{R}\left(\Sigma_{p}\right) \rightarrow \bar{B}_{R}(p)=X$ may not satisfy the assumptions of Theorem A).

In the proof of Theorem 2.2, our main technical lemma is Lemma 2.12. Let $\phi: \Sigma \times\{R\} \rightarrow \Sigma \times\{R\}$ be defined as in Theorem 2.2. We first observe that $\phi$ is an involution. Let $L_{p}(X)=\exp _{p}(\Sigma \times\{R\})=\{x \in X:|p x|=R\}$.

Lemma 2.6. Let $X=\bar{C}_{\kappa}^{R}(\Sigma) / x \sim x^{\prime} \in \operatorname{Alex}^{n}(\kappa)$ be defined as in Theorem 2.2. For any $q \in L_{p}(X)$, if $\tilde{q}_{1} \neq \tilde{q}_{2}$ with $\exp _{p}\left(\tilde{q}_{1}\right)=\exp _{p}\left(\tilde{q}_{2}\right)=q$, then the loop $\exp _{p}\left(\left[\tilde{o} \tilde{q}_{1}\right]\right) \cup \exp _{p}\left(\left[\tilde{o} \tilde{q}_{2}\right]\right)$ forms a local geodesic at $q$. Consequently, $\exp _{p}^{-1}(q)$ contains at most two points.

Proof. It's clear that $\exp _{p}\left(\left[\tilde{o} \tilde{q}_{i}\right]\right)$ are minimal geodesics, $i=1,2$. Let $x_{i} \in X$ be a point on $\exp _{p}\left(\left[\tilde{o} \tilde{q}_{i}\right]\right)$ and $\tilde{x}_{i}=\exp _{p}^{-1}\left(x_{i}\right), i=1,2$. We claim that if $x_{1}, x_{2}$ 
are both close enough to $q$, the geodesic $\left[x_{1} x_{2}\right]$ intersects with $L_{p}(X)$. If not, $\left[x_{1} x_{2}\right] \subset B_{R}(p)$. By the assumption, $\left|x_{1} x_{2}\right|_{X}=\left|\tilde{x}_{1} \tilde{x}_{2}\right|_{\bar{C}_{\kappa}^{R}(\Sigma)}$. Let $x_{1}, x_{2} \rightarrow q$. We get that $\left|x_{1} x_{2}\right|_{X} \rightarrow 0$ and $\left|\tilde{x}_{1} \tilde{x}_{2}\right|_{\bar{C}_{\kappa}^{R}(\Sigma)} \rightarrow\left|\tilde{q}_{1} \tilde{q}_{2}\right|_{\bar{C}_{K}^{R}(\Sigma)}>0$, a contradiction.

Let $a \in\left[x_{1} x_{2}\right] \cap L_{p}(X)$. It remains to show that $a=q$. For $i=1,2$,

$$
\left|x_{i} a\right| \geq|p a|-\left|p x_{i}\right|=|p q|-\left|p x_{i}\right|=\left|x_{i} q\right| .
$$

Thus

$$
\left|x_{1} q\right|+\left|x_{2} q\right| \leq\left|x_{1} a\right|+\left|x_{2} a\right|=\left|x_{1} x_{2}\right|,
$$

which forces both of the above inequalities to be equalities, and thus $a=q$.

As a corollary of Lemma 2.6, we conclude that for $X \in \mathscr{A}_{\kappa}^{R}(\Sigma), \kappa>0$, and $\pi /(2 \sqrt{\kappa})<R<\pi / \sqrt{\kappa}, \operatorname{vol} C_{\kappa}^{R}(\Sigma)$ is not the optimal upper bound for vol $X$; see [Grove and Petersen 1992]. Equivalently, we have:

Corollary 2.7. Assume $X \in \mathscr{A}_{\kappa}^{R}(\Sigma)$ with $\operatorname{vol}(X)=\operatorname{vol} \bar{C}_{\kappa}^{R}(\Sigma)$ and $\kappa>0$. Then $R \leq \pi /(2 \sqrt{\kappa})$ or $R=\pi / \sqrt{\kappa}$. In the second case, $X=C_{\kappa}(\Sigma)$ which is the $k$ suspension of $\Sigma$.

Proof. Assume $\pi /(2 \sqrt{\kappa})<R<\pi / \sqrt{\kappa}$. Let $p \in X$ such that $\Sigma_{p}=\Sigma$. It's clear that $\operatorname{rad}_{p}(X)=R$. We claim that $L_{p}(X)=\{q\}$ has only one point. Then by Lemma 2.6, $\Sigma_{p} \times\{R\}=\exp _{p}^{-1}(q)$ contains at most two points, a contradiction. Let $a \neq b \in L_{p}(X)$. Consider the triangle $\triangle p a b$ and the compared triangle $\widetilde{\triangle} a b \in S_{\kappa}^{2}$. Take $c \in[a b]$ and the corresponding $\tilde{c} \in[\tilde{a} \tilde{b}]$ with $|a c|=|\tilde{a} \tilde{c}|$. By the triangle comparison, $|p c| \geq|\tilde{p} \tilde{c}|>R$, a contradiction. Note that the case where $R=\pi / \sqrt{\kappa}$ follows from Theorem 2.1.

It remains to show that $\phi$ is an isometry. The following lemma plays an important role in the study of the angles in the gluing space $X$.

Lemma 2.8. Let $a, b \in C_{\kappa}(\Sigma)$. Then $\measuredangle a p b=\widetilde{\measuredangle} a p b$ and $\measuredangle p a b=\widetilde{\measuredangle} p a b$.

Proof. The proofs are essentially the same for different $\kappa$. For simplicity, we only give a proof for $\kappa=0$. Note that $\measuredangle a p b=\widetilde{\measuredangle} a p b$ by the definition of $C_{\kappa}(\Sigma)$.

To see $\measuredangle p a b=\widetilde{\measuredangle} p a b$, shortly extend the geodesic [pa] to $a^{\prime}$ and apply the cosine law to the triangles $\triangle a a^{\prime} b, \triangle p a^{\prime} b$, and $\triangle p a b$. We get

$$
\begin{aligned}
\left|a^{\prime} b\right|^{2} & =\left|a a^{\prime}\right|^{2}+|a b|^{2}-2\left|a a^{\prime}\right||a b| \cos \measuredangle a^{\prime} a b, \\
\left|a^{\prime} b\right|^{2} & =\left|p a^{\prime}\right|^{2}+|p b|^{2}-2\left|p a^{\prime}\right||p b| \cos \measuredangle a p b \\
& =\left(|p a|+\left|a a^{\prime}\right|\right)^{2}+|p b|^{2}-2\left(|p a|+\left|a a^{\prime}\right|\right)|p b| \cos \measuredangle a p b, \\
|a b|^{2} & =|p a|^{2}+|p b|^{2}-2|p a||p b| \cos \measuredangle a p b .
\end{aligned}
$$


Calculating $(2-1)+(2-3)-(2-2)$, we get

$$
\begin{aligned}
0 & =|a b| \cos \widetilde{\measuredangle} a^{\prime} a b+|p a|-|p b| \cos \measuredangle a p b \\
& \geq|a b| \cos \measuredangle a^{\prime} a b+|p a|-|p b| \cos \measuredangle a p b \\
& =-|a b| \cos \measuredangle p a b+|p a|-|p b| \cos \measuredangle a p b .
\end{aligned}
$$

Since $\measuredangle p a b \geq \widetilde{\measuredangle} p a b$ and $\measuredangle a p b=\widetilde{\measuredangle} a p b$, the above inequality implies

$$
\begin{aligned}
|p a| & \leq|a b| \cos \measuredangle p a b+|p b| \cos \measuredangle a p b \\
& \leq|a b| \cos \widetilde{\measuredangle} p a b+|p b| \cos \widetilde{\measuredangle} a p b=|p a|,
\end{aligned}
$$

which forces $\measuredangle p a b=\widetilde{\measuredangle} p a b$.

Corollary 2.9. Let $x, y \in X$ be two points. If $[x y] \cap L_{p}(X) \neq \varnothing$, then either $[x y] \subset L_{p}(X)$ or $[x y] \cap L_{p}(X)$ is finite.

Proof. Let $x \notin L_{p}(X)$. We show that $[x y] \cap L_{p}(X)$ is finite. Let $a \in[x y] \cap L_{p}(X)$ be the accumulation point which is closest to $x$. Clearly $a \neq x$ since $x \notin L_{p}(X)$. Thus there is a geodesic segment $[b a]$ of $[x y]$ with $[b a]-\{a\} \subset B_{R}(p)$. Since $|p b|<|p a|=R$, by Lemma 2.8,

$$
\measuredangle p a b=\widetilde{\measuredangle} p a b<\frac{\pi}{2} .
$$

On the other hand, because there are $a_{i} \in[x y] \cap L_{p}(X)$ with $a_{i} \rightarrow a$ as $i \rightarrow \infty$ and $|p a|=\left|p a_{i}\right|=R$, by the first variation formula, we get

$$
\measuredangle p a y=\frac{\pi}{2} .
$$

Therefore $\pi=\measuredangle p a b+\measuredangle p a y<\pi$, a contradiction.

As another corollary, we prove Theorem 2.2 for the special case $\kappa>0$ and $R=\pi /(2 \sqrt{\kappa})$.

Corollary 2.10. Theorem 2.2 holds for the case $\kappa>0$ and $R=\pi /(2 \sqrt{\kappa})$.

Proof. Let $x, y \in L_{p}(X), \tilde{x}_{1}, \tilde{x}_{2}, \tilde{y}_{1}, \tilde{y}_{2} \in \Sigma \times\{R\}$ with $\exp _{p}\left(\tilde{x}_{1}\right)=\exp _{p}\left(\tilde{x}_{2}\right)=x$, $\exp _{p}\left(\tilde{y}_{1}\right)=\exp _{p}\left(\tilde{y}_{2}\right)=y$. We will show that $\left|\tilde{x}_{1} \tilde{y}_{1}\right|_{\bar{C}_{\kappa}^{R}(\Sigma)}=\left|\tilde{x}_{2} \tilde{y}_{2}\right|_{\bar{C}_{\kappa}^{R}(\Sigma)}$. Assume $\left|\tilde{x}_{1} \tilde{y}_{1}\right|_{\bar{C}_{\kappa}^{R}(\Sigma)}>\left|\tilde{x}_{2} \tilde{y}_{2}\right|_{\bar{C}_{\kappa}^{R}(\Sigma)}$. Then there is a point $a \notin L_{p}(X) \operatorname{ctake}_{\exp _{p}^{-1}(a) \text { close }}$ to $\left.x_{1}\right)$ such that $[a y] \cap L_{p}(X)$ contains a point $b \neq y$. Because $\exp _{p}$ is distancenonincreasing and $\Sigma \times\{\pi /(2 \sqrt{\kappa})\}$ is totally geodesic, $[b y] \subset L_{p}(X)$, which contradicts Corollary 2.9 .

Let $\operatorname{Fix}(\phi)=\{\tilde{x} \in \Sigma \times\{R\}: \phi(\tilde{x})=\tilde{x}\}$ be the fixed points set. Let $L_{p}^{1}(X)=$ $\exp _{p}(\operatorname{Fix}(\phi))$ denote the image. Due to Lemma 2.6, let $L_{p}^{2}(X)=L_{p}(X)-L_{p}^{1}(X)$ denote the points that are identified from exactly two points, that is, for any

$$
x \in L_{p}^{2}(X),
$$


$\exp ^{-1}(x)=\left\{\tilde{x}^{+}, \tilde{x}^{-}\right\}$contains exactly two points.

In the rest of the proof of Theorem 2.2, by Corollaries 2.9 and 2.10 and their proofs, we can always assume $R<\pi /(2 \sqrt{\kappa})$ for $\kappa>0$ and that for any $x, y \in X$, $[x y] \cap L_{p}(X)$ is finite if it is not empty. Moreover, the following corollary shows that $] x y\left[\cap L_{p}(X) \subset L_{p}^{2}(X)\right.$, where $] x y$ [ denotes the geodesic connecting $x, y$ without the end points.

Corollary 2.11. Let the assumption be as in Theorem 2.2. Assume $R<\pi /(2 \sqrt{\kappa})$ when $\kappa>0$. For any $x, y \in X$, if $q \in] x y\left[\cap L_{p}(X), q \in L_{p}^{2}(X)\right.$.

Proof. Without losing generality, we assume $x, y \notin L_{p}(X)$ and $] x y\left[\cap L_{p}(X)=\{q\}\right.$. If $q \in L_{p}^{1}(X)$, by Lemma 2.8, $\measuredangle x q p=\widetilde{\measuredangle} x q p<\pi / 2$ and $\measuredangle y q p=\widetilde{\measuredangle} y q p<\pi / 2$. Thus $\measuredangle x q p+\measuredangle y q p<\pi$, which contradicts the fact that $[x y]$ is a geodesic.

Now we are ready to prove our main technical lemma. Let $x \in L_{p}^{2}(X)$ and $\left\{\tilde{x}^{+}, \tilde{x}^{-}\right\}=\exp _{p}^{-1}(x)$ denote the preimage. Then there are exactly two geodesics $\exp _{p}\left(\left[\tilde{o} \tilde{x}^{+}\right]\right), \exp _{p}\left(\left[\tilde{o} \tilde{x}^{-}\right]\right)$connecting $x$ to $p$. To distinguish geodesics and angles, we use the following notation.

- Let $\left[p x^{+}\right]$and $\left[p x^{-}\right]$denote $\exp _{p}\left(\left[\tilde{o} \tilde{x}^{+}\right]\right)$and $\exp _{p}\left(\left[\tilde{o} \tilde{x}^{-}\right]\right)$respectively. In addition, for $y \in L_{p}^{2}(X)$ and $\exp _{p}^{-1}(y)=\left\{\tilde{y}^{+}, \tilde{y}^{-}\right\}$:

- let $\left[x^{ \pm} y^{ \pm}\right]$denote $\exp _{p}\left(\left[\tilde{x}^{ \pm} \tilde{y}^{ \pm}\right]\right)$;

- let $\left|x^{ \pm} y^{ \pm}\right|$denote the length of the geodesics $\left[x^{ \pm} y^{ \pm}\right]$;

- let $\measuredangle x^{ \pm} p y^{ \pm}$denote the angle between $\left[p x^{ \pm}\right]$and $\left[p y^{ \pm}\right]$at $p$;

- let $\measuredangle p x^{ \pm} y^{ \pm}$denote the angle between $\left[p x^{ \pm}\right]$and $\left[x^{ \pm} y^{ \pm}\right]$at $x$.

Lemma 2.12. Let the assumptions be as in Theorem 2.2. Assume $R<\pi /(2 \sqrt{\kappa})$ when $\kappa>0$. Then, for any $\tilde{x} \neq \tilde{y} \in \Sigma \times\{R\}$ with $|\tilde{x} \tilde{y}|$ sufficiently small,

$$
\left|\frac{|\phi(\tilde{x}) \phi(\tilde{y})|}{|\tilde{x} \tilde{y}|}-1\right| \leq 20|\tilde{x} \tilde{y}| .
$$

Proof. For simplicity, we give a proof for the case $\kappa=0$. The other cases can be carried out similarly. Throughout the proof, we will frequently use lemmas 2.6 and 2.8 and Corollary 2.11 without mentioning it. We will also assume that for any $a, b \in X,[a b] \cap L_{p}(X)$ is finite if it is not empty.

Clearly, $\phi$ preserves the distance when $x$ and $y$ are both in $L_{p}^{1}(X)$. Let

$$
x \in L_{p}^{2}(X), \quad y \in L_{p}(X)
$$

(if $y \in L_{p}^{1}(X), \tilde{y}^{+}=\tilde{y}^{-}$will denote the same point and the argument will still go through). Because $[x y] \cap L_{p}(X)$ is finite, not losing generality, assume $[x y]=$ 


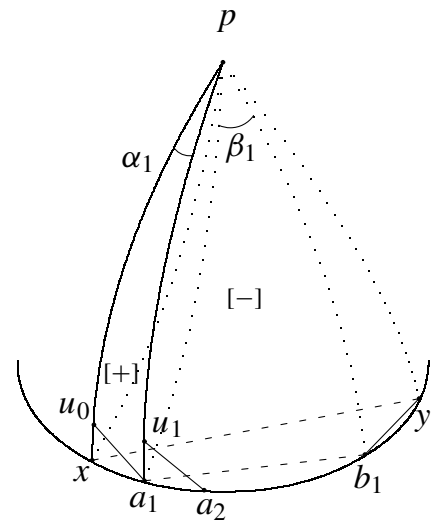

Figure 3

$\left[x^{-} y^{-}\right]$. Thus $\measuredangle x^{-} p y^{-} \leq \measuredangle x^{+} p y^{+}$. Let $\beta_{0}=\measuredangle x^{-} p y^{-}$. Since $\left|x^{-} y^{-}\right|=2 R \sin \frac{\beta_{0}}{2}$ and $\left|x^{+} y^{+}\right|=2 R \sin \left(\measuredangle x^{+} p y^{+} / 2\right)$, it's sufficient to show that

$$
10 \beta_{0}^{2}+\beta_{0} \geq \measuredangle x^{+} p y^{+} \text {. }
$$

Take $u_{0} \in\left[p x^{+}\right]$with $\left|u_{0} x^{+}\right|=\epsilon$. Let $\left[u_{0} y\right]$ be a geodesic. If

$$
\left[\left(u_{0} y\right)\right] \cap L_{p}(X) \neq \varnothing
$$

let $a_{1}(\neq y)$ and $b_{1}\left(b_{1}\right.$ can be $\left.y\right)$ be the first and second intersection points in $\left[u_{0} y\right] \cap L_{p}(X)$ along the direction $\uparrow^{y} u_{0}$ (see Figure 3). Assign \pm to $\exp _{p}^{-1}\left(a_{1}\right)$, $\exp _{p}^{-1}\left(b_{1}\right)$ such that $\measuredangle p a_{1}^{+} u_{0}<\pi / 2$. Let $\alpha_{1}=\measuredangle x^{+} p a_{1}^{+}$and $\beta_{1}=\measuredangle a_{1}^{-} p b_{1}^{-}$. In the case of $\left[\left(u_{0} y\right)\right] \cap L_{p}(X)=\varnothing$, we take $b_{1}=a_{1}=y$ and $\beta_{1}=0$.

Because $\left[u_{0} a_{1}^{+}\right] *\left[a_{1}^{-} b_{1}^{-}\right] *\left[b_{1}^{+} y\right]$ is a minimal geodesic, by triangle inequality,

$$
\left|u_{0} x\right|+|x y| \geq\left|u_{0} a_{1}^{+}\right|+\left|a_{1}^{-} b_{1}^{-}\right|+\left|b_{1} y\right| .
$$

This implies

$$
\epsilon+2 R \sin \frac{\beta_{0}}{2} \geq\left|u_{0} a_{1}^{+}\right|+2 R \sin \frac{\beta_{1}}{2} .
$$

Applying the cosine law (the form in Lemma 4.7(5)) in $\triangle p u_{0} a_{1}$ with the angle $\measuredangle u_{0} p a_{1}^{+}=\alpha_{1}$, we get that

$$
\left|u_{0} a_{1}^{+}\right|=\sqrt{\epsilon^{2}+4 R(R-\epsilon) \sin ^{2} \frac{\alpha_{1}}{2}} \geq 2(R-\epsilon) \sin \frac{\alpha_{1}}{2} .
$$

Thus

$$
\epsilon+2 R \sin \frac{\beta_{0}}{2} \geq 2(R-\epsilon) \sin \frac{\alpha_{1}}{2}+2 R \sin \frac{\beta_{1}}{2}
$$


If $\left[\left(u_{0} y\right)\right] \cap L_{p}(X)=\varnothing$, we stop here. If $\left[\left(u_{0} y\right)\right] \cap L_{p}(X) \neq \varnothing$, we proceed with $u_{1} \in\left[p a_{1}^{+}\right]$and $\left|u_{1} a_{1}\right|=\epsilon$. Let $\left[u_{1} b_{1}\right]$ be a geodesic. Again, if $\left[\left(u_{1} b_{1}\right)\right] \cap L_{p}(X) \neq$ $\varnothing$, let $a_{2}(\neq y)$ and $b_{2}$ (can be $\left.b_{1}\right)$ be the first and second intersection points in $\left[u_{1} b_{1}\right] \cap L_{p}(X)$ along the direction $\uparrow_{u_{1}}^{b_{1}}$. Assign \pm to $\exp _{p}^{-1}\left(a_{2}\right), \exp _{p}^{-1}\left(b_{2}\right)$ such that $\measuredangle p a_{2}^{+} u_{1}<\pi / 2$. Let $\alpha_{2}=\measuredangle a_{1}^{+} p a_{2}^{+}$and $\beta_{2}=\measuredangle a_{2}^{-} p b_{2}^{-}$. If $\left[\left(u_{1} b_{1}\right)\right] \cap L_{p}(X)=\varnothing$, $a_{2}=b_{2}=b_{1}, \beta_{2}=0$, and we stop the process. Proceed inductively until $\left[\left(u_{N} b_{N}\right)\right] \cap$ $L_{p}(X)=\varnothing$, which yields that $a_{N+1}=b_{N+1}=b_{N}$ and $\beta_{N+1}=0$. We claim that $N$ is finite, and, moreover,

$$
(N+1) \epsilon<5 R \beta_{0}^{2} .
$$

For each $0 \leq i \leq N$, we have

$$
\begin{gathered}
\epsilon+2 R \sin \frac{\beta_{i}}{2} \geq\left|u_{i} a_{i+1}^{+}\right|+2 R \sin \frac{\beta_{i+1}}{2}, \\
\epsilon+2 R \sin \frac{\beta_{i}}{2} \geq 2(R-\epsilon) \sin \frac{\alpha_{i+1}}{2}+2 R \sin \frac{\beta_{i+1}}{2},
\end{gathered}
$$

where $\alpha_{i}=\measuredangle a_{i}^{+} p a_{i+1}^{+}, \beta_{i}=\measuredangle a_{i}^{-} p b_{i}^{-}$. Summing up (2-9) for $i=0,1, \ldots, N$ and applying (2-7), we get

$$
\begin{aligned}
5 R \beta_{0}^{2}+2 R \sin \frac{\beta_{0}}{2} & \geq(N+1) \epsilon+2 R \sin \frac{\beta_{0}}{2} \\
& \geq 2(R-\epsilon) \sum_{i=1}^{N} \sin \frac{\alpha_{i}}{2} \geq 2(R-\epsilon) \sin \frac{\sum_{i=1}^{N} \alpha_{i}}{2} \\
& \geq 2(R-\epsilon) \sin \frac{\measuredangle x^{+} p b_{N}}{2} .
\end{aligned}
$$

Since $b_{N} \rightarrow b_{1} \rightarrow y^{+}$when taking $\epsilon \rightarrow 0$, (2-4) follows.

It remains to show (2-7). A sum of (2-8) for $i=0,1, \ldots, N$ indicates that the upper bound of $N$ relies on an estimate of $\left|u_{i} a_{i+1}^{+}\right|$in terms of $\epsilon$ and $\beta_{i+1}$. Noting that $a_{i+1}=\left[u_{i} b_{i+1}\right] \cap\left(\left[p a_{i+1}^{+}\right] *\left[p a_{i+1}^{-}\right]\right)$and $\left[p a_{i+1}^{+}\right] *\left[p a_{i+1}^{-}\right]$is a local geodesic at $a_{i+1}$, we have $\measuredangle p a_{i+1}^{+} u_{i}=\measuredangle p a_{i+1}^{-} b_{i+1}=\pi / 2-\beta_{i+1} / 2$. Applying the cosine law in triangle $\triangle p u_{i} a_{i+1}^{+}$, we get

that is,

$$
(R-\epsilon)^{2}=R^{2}+\left|u_{i} a_{i+1}^{+}\right|^{2}-2 R\left|u_{i} a_{i+1}^{+}\right| \sin \frac{\beta_{i+1}}{2},
$$

$$
\left|u_{i} a_{i+1}^{+}\right|^{2}-2 R \sin \frac{\beta_{i}}{2}\left|u_{i} a_{i+1}^{+}\right|+R \epsilon-\epsilon^{2}=0 .
$$

Solving for $\left|u_{i} a_{i+1}^{+}\right|$and taking into account that $\epsilon>0$ is small, we have

$$
\left|u_{i} a_{i+1}^{+}\right| \geq R \sin \frac{\beta_{i+1}}{2}-\sqrt{\left(R \sin \frac{\beta_{i+1}}{2}\right)^{2}-\left(R \epsilon-\epsilon^{2}\right)}>\frac{\epsilon}{4 \sin \left(\beta_{i+1} / 2\right)} .
$$


Note that $\beta_{i}$ is decreasing, which is implied by (2-8) and $\left|u_{i} a_{i+1}^{+}\right|>\left|u_{i} a_{i}^{+}\right|=\epsilon$. We get

$$
\left|u_{i} a_{i+1}^{+}\right|>\frac{\epsilon}{4 \sin \left(\beta_{0} / 2\right)} .
$$

Plugging (2-10) into (2-8), we get

$$
\epsilon+2 R \sin \frac{\beta_{i}}{2}>\frac{\epsilon}{4 \sin \left(\beta_{0} / 2\right)}+2 R \sin \frac{\beta_{i+1}}{2} .
$$

Summing up (2-11) for $i=0,1, \ldots, N$, we get

$$
(N+1) \epsilon+2 R \sin \frac{\beta_{0}}{2}>(N+1) \frac{\epsilon}{4 \sin \left(\beta_{0} / 2\right)} .
$$

Therefore

$$
(N+1) \epsilon<\frac{8 R \sin ^{2}\left(\beta_{0} / 2\right)}{1-4 \sin \left(\beta_{0} / 2\right)}<5 R \beta_{0}^{2} .
$$

Proof of Theorem 2.2, assuming $R<\pi /(2 \sqrt{\kappa})$ when $\kappa>0$. By Lemma 2.12, $\phi$ is a continuous involution and thus a homeomorphism. It reduces to show that $\phi: \Sigma \times\{R\} \rightarrow \Sigma \times\{R\}$ preserves the length of any curve $c:[0,1] \rightarrow \Sigma \times\{R\}$. Given $\delta, \epsilon>0$, we may assume a partition $P: 0=t_{0}<t_{1}<\cdots<t_{N}=1$ with $\left|c\left(t_{i}\right) c\left(t_{i+1}\right)\right| \leq \delta$ such that the length of the curve satisfies

$$
L(c)<\sum_{i=0}^{N-1}\left|c\left(t_{i}\right) c\left(t_{i+1}\right)\right|+\frac{\epsilon}{2}, \quad L(\phi(c))<\sum_{i=0}^{N-1}\left|\phi\left(c\left(t_{i}\right)\right) \phi\left(c\left(t_{i+1}\right)\right)\right|+\frac{\epsilon}{2} .
$$

Then

$$
\begin{aligned}
|L(c)-L(\phi(c))| & \leq \sum_{i=0}^{N-1}|| c\left(t_{i}\right) c\left(t_{i+1}\right)|-| \phi\left(c\left(t_{i}\right)\right) \phi\left(c\left(t_{i+1}\right)\right)||+\epsilon \\
& \leq \sum_{i=0}^{N-1} 20\left|c\left(t_{i}\right) c\left(t_{i+1}\right)\right|^{2}+\epsilon \\
& \leq 20 \delta \sum_{i=0}^{N-1}\left|c\left(t_{i}\right) c\left(t_{i+1}\right)\right|+\epsilon \\
& \leq 20 \delta L(c)+\epsilon .
\end{aligned}
$$

Since $\epsilon>0$ and $\delta>0$ can be chosen arbitrarily small, we get the desired result.

Completion of Proof of Theorem B. By Theorems 2.1 and 2.2, we identify $X$ with $\bar{C}_{\kappa}^{R}\left(\Sigma_{p}\right) / x \sim \phi(x)$. We show that the metric on $X$ coincides with the metric induced from the identification $x \sim \phi(x)$. It's equivalent to show that

$$
\exp _{p}: \bar{C}_{\kappa}^{R}\left(\Sigma_{p}\right) \rightarrow X
$$


preserves lengths of geodesics. Let $\gamma \subset \bar{C}_{\kappa}^{R}\left(\Sigma_{p}\right)$ be a geodesic and $\sigma=f(\gamma)$. Since $L(\gamma) \geq L(\sigma)$, it remains to show that $L(\sigma) \geq L(\gamma)$. Because either $\gamma \subset \Sigma \times\{R\}$ or $\gamma \cap(\Sigma \times\{R\})$ has at most two points, we need only check for the case $\gamma \subset \Sigma \times\{R\}$, that is, $\sigma \subset L_{p}(X)$. For any $\epsilon>0$, let $\left\{x_{i}\right\}_{i=0}^{2 N+1} \subset \sigma$ be an $\epsilon$-partition and

$$
L(\sigma)=\lim _{\epsilon \rightarrow 0} \sum_{i=0}^{2 N}\left|x_{i} x_{i+1}\right| .
$$

Let $a_{i} \in \gamma$ so that $\exp _{p}\left(a_{i}\right)=x_{i}$. Choose $b_{2 k} \in C_{\kappa}^{R}(\Sigma), k=0,1, \ldots, N$, with $\left|a_{2 k}-b_{2 k}\right|<\epsilon^{4}$. Let $b_{2 k+1}=a_{2 k+1}$ for $k=0,1, \ldots, N$ and $y_{i}=\exp _{p}\left(b_{i}\right)$ for $i=0,1, \ldots 2 N+1$. Then $\left|y_{i}-x_{i}\right| \leq\left|b_{i}-a_{i}\right|<\epsilon^{4}$ and thus

$$
L(\sigma)=\lim _{\epsilon \rightarrow 0} \sum_{i=0}^{2 N}\left|y_{i} y_{i+1}\right| .
$$

We claim that $\left[y_{i} y_{i+1}\right] \cap L_{p}(X)$ is either $y_{i}$ or $y_{i+1}$. By Corollary 2.9, let

$$
u, v \in\left[y_{i} y_{i+1}\right] \cap L_{p}(X)
$$

and there is no crossing point in between. Without losing generality, we assume $y_{i} \notin L_{p}(X)$ and $\left|y_{i} u\right|<\left|y_{i} v\right|$. Let $\left[u^{-} v^{-}\right] \subset\left[y_{i} y_{i+1}\right]$. Because the involution $\phi$ is an isometry (Theorem 2.2), $L\left(\left[u^{+} v^{+}\right]\right)=L\left(\left[u^{-} v^{-}\right]\right)$. Thus $\left[y_{i} u\right] \cup\left[u^{+} v^{+}\right] \neq$ $\left[y_{i} u\right] \cup\left[u^{-} v^{-}\right]$is also a geodesic, which yields a bifurcation of geodesics.

By the claimed property, we have that $\left|y_{i} y_{i+1}\right|=\left|b_{i} b_{i+1}\right|$. Since $\sum_{i=0}^{2 N}\left|b_{i} b_{i+1}\right| \geq$ $L(\gamma)$, we have

$$
L(\sigma)=\lim _{\epsilon \rightarrow 0} \sum_{i=0}^{2 N}\left|y_{i} y_{i+1}\right|=\lim _{\epsilon \rightarrow 0} \sum_{i=0}^{2 N}\left|b_{i} b_{i+1}\right| \geq L(\gamma) .
$$

It remains to show that for $\Sigma \in \operatorname{Alex}^{n-1}(1)$, if $\phi: \Sigma \times\{R\} \rightarrow \Sigma \times\{R\}$ is an isometric involution, $X=\bar{C}_{\kappa}^{R}(\Sigma) /(x \sim \phi(x)) \in \operatorname{Alex}^{n}(\kappa)$.

Case 1. Assume $\partial \Sigma=\varnothing$. Take two copies of $\bar{C}_{\kappa}^{R}(\Sigma)$, marked as $\bar{C}_{\kappa}^{R}(\Sigma)_{1}$ and $\bar{C}_{\kappa}^{R}(\Sigma)_{2}$, whose vertices are $p_{1}$ and $p_{2}$, respectively. Gluing along their boundaries by $\phi$, we obtain a double space $\widehat{X}=\bar{C}_{\kappa}^{R}(\Sigma)_{1} \cup_{\phi} \bar{C}_{\kappa}^{R}(\Sigma)_{2}$. By the gluing theorem [Petrunin 1997], $\widehat{X} \in \operatorname{Alex}^{n}(\kappa)$.

Now we extend the isometric $\mathbb{Z}_{2}$-action by $\phi$ on $\Sigma$ to an isometric $\hat{\mathbb{Z}}_{2}$-action on $\widehat{X}$ such that $X=\widehat{X} / \hat{\mathbb{Z}}_{2}$, and thus $X \in \operatorname{Alex}^{n}(\kappa)$. For any $u \in \bar{C}_{\kappa}^{R}(\Sigma)_{1}$, extend the geodesic $\left[p_{1} u\right]_{\bar{C}_{\kappa}^{R}(\Sigma)_{1}}$ to $u_{1} \in(\Sigma \times\{R\})_{1}$. Let $\hat{\phi}(u)$ be the point on the geodesic $\left[p_{2} \phi\left(u_{1}\right)\right]_{\bar{C}_{\kappa}^{R}(\Sigma)_{2}}$ such that $\left|p_{2} \hat{\phi}(u)\right|=\left|p_{1} u\right|$ (so $\left.\hat{\phi}: \bar{C}_{\kappa}^{R}(\Sigma)_{1} \rightarrow \bar{C}_{\kappa}^{R}(\Sigma)_{2}\right)$. Switching the roles of $\bar{C}_{\kappa}^{R}(\Sigma)_{1}$ and $\bar{C}_{\kappa}^{R}(\Sigma)_{2}$, we extend $\phi$ to an isometric involution $\hat{\phi}$ : $\bar{C}_{\kappa}^{R}(\Sigma)_{2} \rightarrow \bar{C}_{\kappa}^{R}(\Sigma)_{1}$. Clearly, $\hat{\phi}: \hat{X} \rightarrow \widehat{X}$ is an isometric involution such that $X=\widehat{X} / \hat{\phi}$. 
Case 2. Assume $\partial \Sigma \neq \varnothing$. Let $\hat{\Sigma}=\Sigma^{+} \cup \Sigma^{-}$denote the double of $\Sigma$. We first extend the isometric involution $\phi$ on $\Sigma$ to $\hat{\phi}: \hat{\Sigma} \rightarrow \hat{\Sigma}$ by $\hat{\phi}\left(x_{ \pm}\right)=\phi(x)_{\mp}$, where $x_{+}=x_{-} \in \Sigma$. We then define another isometric involution $\psi: \hat{\Sigma} \rightarrow \hat{\Sigma}$ by the reflection on $\partial \Sigma, \psi\left(x_{ \pm}\right)=x_{\mp}$. Then $\hat{\psi}\left(\hat{\phi}\left(x_{ \pm}\right)\right)=\hat{\psi}\left(\phi(x)_{ \pm}\right)=\phi(x)_{\mp}=\hat{\phi}\left(x_{\mp}\right)=$ $\hat{\phi}\left(\hat{\psi}\left(x_{ \pm}\right)\right)$. This implies that $\hat{\Sigma}$ admits a $\mathbb{Z}_{2} \oplus \mathbb{Z}_{2}$-action. Clearly, the $\mathbb{Z}_{2} \oplus \mathbb{Z}_{2^{-}}$ action extends uniquely to an isometric $\mathbb{Z}_{2} \oplus \mathbb{Z}_{2}$-action on $\bar{C}_{\kappa}^{r}(\hat{\Sigma})$. By Case 1 , we extend only the $\hat{\phi}$-action to $\widehat{X}$ such that $\bar{C}_{\kappa}^{r}(\hat{\Sigma}) / x \sim \hat{\phi}(x) \in \operatorname{Alex}^{n}(\kappa)$. Then $X=\left[\bar{C}_{\kappa}^{r}(\hat{\Sigma}) / x \sim \hat{\phi}(x)\right] / \hat{\psi} \in \operatorname{Alex}^{n}(\kappa)$.

By Theorem B, the isometric classification of $X \in \mathscr{A}_{\kappa}^{r}(\Sigma)$ with relatively maximum volume reduces to the isometric classification of all $(n-1)$-dimensional Alexandrov spaces $\Sigma$ with curv $\geq 1$ and the equivariant isometric $\mathbb{Z}_{2}$-actions on $\Sigma$. For $n=2$, one easily gets a complete list:

Corollary 2.13. Any 2-dimensional compact Alexandrov space with curv $\geq \kappa$ and relatively maximum volume is isometric to one of the following:

$$
\bar{C}_{\kappa}^{r}\left(S_{\theta}^{1}\right) / \phi_{i} \quad(i=1,2,3) \quad \text { or } \quad \bar{C}_{\kappa}^{r}([0, \theta]) / \psi_{i} \quad(i=1,2),
$$

where $S_{\theta}^{1}$ denotes a circle of length $2 \theta$ with $0<\theta \leq \pi$ and $\phi_{i}: S_{\theta}^{1} \rightarrow S_{\theta}^{1}$ (respectively $\left.\psi_{i}:[0, \theta] \rightarrow[0, \theta]\right)$ is trivial, a reflection or the antipodal map respectively for $i=1,2$ and 3 (respectively $i=1$ and 2 ).

Example 2.14 (cf. [Grove and Petersen 1992]). Let $Z=\mathbb{D}^{2}$ be a 2-dimensional flat unit disk. Then $\partial Z=\mathbb{S}^{1}(1)$ is a unit circle. Let $\phi: \partial Z \rightarrow \partial Z$ be a one-to-one map and $X=\mathbb{D}^{2} / x \sim \phi(x)$ the glued space via identification $z \sim \phi(z)$. By Theorem B, $X$ is an Alexandrov space if and only if $\phi$ is an isometric involution, that is, $\phi$ is a reflection, antipodal map, or identity, where $X$ is homeomorphic to $\mathbb{S}^{2}, \mathbb{R} P^{2}$, and $\mathbb{D}^{2}$, respectively.

Example 2.15. Consider a 2-dimensional simplex. We identify points on each side via a reflection about the mid point. Then we get a tetrahedron, in which one vertex is glued from the three vertices of the simplex.

\section{Proof of Theorem C: Relatively almost maximum volume}

In the proof of Theorem $\mathrm{C}$, we need the following result.

Theorem 3.1 [Bredon 1972, Theorem 5.5]. Let $M$ be a $G$-manifold. $G$ is a finite group. Assume that, for a given prime $p$ and all p-subgroups, $P \subseteq G$ satisfies

$$
\left.H_{i}\left(M^{P} ; \mathbb{Z}_{p}\right)=0, \quad i \leq q \text { (including } P=\{e\}\right) .
$$

Then $H_{i}\left(M / G ; \mathbb{Z}_{p}\right)=0$ for all $i \leq q$. Moreover, if this holds for all prime $p$ and $H_{i}(M ; \mathbb{Z})=0$ for $i \leq q$, then $H_{i}(M / G ; \mathbb{Z})=0$ for $i \leq q$. 
Proof of Theorem $C$. We first show that if $X \in \mathscr{A}_{\kappa}^{r}(\Sigma)$ with vol $X=v(\Sigma, \kappa, r), X$ is homeomorphic to $S^{n}$ or $\mathbb{C} P^{n}$.

By Theorem B, $X$ is isometric to $\left.\bar{C}_{\kappa}^{R}(\Sigma)\right) / x \sim \phi(x)$ and $\phi: \Sigma \rightarrow \Sigma$ is an isometric involution. To determine the homeomorphism type of $X$, we consider the double space $\left.\left.\widehat{X}=\bar{C}_{\kappa}^{R}(\Sigma)\right)^{+} \cup_{\phi} \bar{C}_{\kappa}^{R}(\Sigma)\right)^{-}$. As seen in the proof of Theorem B, $\hat{X} \in \operatorname{Alex}^{n}(\kappa)$ and $\phi$ extends an isometric $\mathbb{Z}_{2}$-action on $\hat{X}$ such that $\hat{X} / \mathbb{Z}_{2}$.

We claim that $\hat{X}$ is a homeomorphism sphere. First, $\hat{X}$ is a topological manifold if every point $\left.\hat{q} \in \partial \bar{C}_{\kappa}^{R}(\Sigma)\right) \hookrightarrow \hat{X}$ is a manifold point. According to [Wu 1997], a point $x$ in an Alexandrov space is a manifold point if and only if $\Sigma_{x}$ is simply connected. Because $\Sigma_{\hat{q}}$ is a suspension of $\Sigma_{\hat{q}}(\Sigma), \hat{q}$ is a manifold point. By the Poincaré conjecture (in all dimensions), our claim reduces to the following: $\hat{X}$ is an integral homotopy sphere. Because $\hat{X}$ is a suspension, $\hat{X}$ is simply connected, and thus it suffices to show that $\hat{X}$ is a homology sphere. Because $\bar{C}_{\kappa}^{R}(\Sigma)$ is contractible, from the Mayer-Vietoris exact sequence of $\left(\bar{C}_{\kappa}^{R}(\Sigma)^{+}, \bar{C}_{\kappa}^{R}(\Sigma)^{-}\right)$, it is easy to see that $\hat{X}$ is an integral homology sphere.

If the $\mathbb{Z}_{2}$-action is free, $X=\hat{X} / \mathbb{Z}_{2}$ is homeomorphic to $\mathbb{R} P^{n}$. Otherwise, $X$ is a simply connected topological manifold (the induced map, $\pi_{1}(\hat{X}) \rightarrow \pi_{1}(X)$ is an onto map). Again, it suffices to show that $X$ is an integral homology sphere. By the Smith theorem, the $\mathbb{Z}_{2}$-fixed point set $\hat{X}^{\mathbb{Z}_{2}}$ is a $\mathbb{Z}_{2}$-homology sphere. By now we can apply Theorem 3.1 to conclude the claim.

We prove Theorem $C$ by contradiction; assume a sequence $X_{i} \in \mathscr{A}_{\kappa}^{r}(\Sigma)$ such that vol $X_{i}>\operatorname{vol} C_{\kappa}^{R}(\Sigma)-\epsilon_{i}\left(\epsilon_{i}=i^{-1}\right)$ and none of $X_{i}$ is homeomorphic to $S^{n}$ or $\mathbb{R} P^{n}$. Without loss of generality, we may assume that

$$
\left(X_{i}, p_{i}\right) \stackrel{d_{G H}}{\longrightarrow}(X, p) \in \operatorname{Alex}^{n}(\kappa),
$$

where $X_{i}=\bar{B}_{r}\left(p_{i}\right)$. By Perelman's stability theorem [Kapovitch 2007; Perelman 1991], $X_{i}$ is homeomorphic to $X$ for $i$ large. In particular, $X$ is a topological manifold. We claim that $X \in \mathscr{A}_{\kappa}^{r}\left(\Sigma_{p}\right)$ satisfies vol $X=v\left(\Sigma_{p}, \kappa, r\right)$. By the above, we then conclude that $X$ is homeomorphic to $S^{n}$ or $\mathbb{R} P^{n}$, and thus $X_{i}$ is homeomorphic to $X$ for $i$ large, a contradiction.

To see the claim,

$$
\operatorname{vol} X=\lim _{i \rightarrow \infty} \operatorname{vol} X_{i}=\lim _{i \rightarrow \infty}\left(\operatorname{vol} C_{\kappa}^{R}(\Sigma)-\epsilon_{i}\right)=\operatorname{vol} C_{\kappa}^{R}(\Sigma) .
$$

On the other hand, we shall construct a distance-nonincreasing map, $\phi: \Sigma \rightarrow \Sigma_{p}$. Consequently, vol $\Sigma_{p} \leq \operatorname{vol} \Sigma$ and thus

$$
\operatorname{vol} X \leq \operatorname{vol} C_{\kappa}^{R}\left(\Sigma_{p}\right) \leq \operatorname{vol} C_{\kappa}^{R}(\Sigma) \leq \operatorname{vol} X .
$$

Let $A=\left\{v_{i}\right\} \subset \Sigma$ be a countable dense subset and $f_{i}:\left(X_{i}, p_{i}\right) \rightarrow(X, p)$ a sequence of $\epsilon_{i}$-Gromov-Hausdorff approximations, $\epsilon_{i} \rightarrow 0$. For $v_{1}$, the sequence 
$\left\{f_{i}\left(g \exp _{p_{i}} v\right)\right\} \subset X$ contains a converging subsequence

$$
f_{i_{1}}\left(g \exp _{p_{i_{1}}} q(v)\right) \rightarrow x_{1} \in X .
$$

Then $\left[p x_{1}\right]=w_{1} \in \Sigma_{p}$ (which may not be unique). We define $\phi\left(v_{1}\right)=w_{1}$. For $v_{2}$ and $\left\{f_{i_{1}}\right\}$, repeating the above, we obtain $w_{2} \in \Sigma_{p}$ and define $\phi\left(v_{2}\right)=w_{2}$. Iterating this process, we define a map $\phi: A \rightarrow \Sigma_{p}, \phi\left(v_{i}\right)=w_{i}$. It is easy to check that $\phi$ is distance-nonincreasing and thus $\phi$ extends uniquely to a distance-nonincreasing map from $\Sigma$ to $\Sigma_{p}$.

\section{Proof of Theorem D:}

\section{Pointed Bishop-Gromov relative volume comparison}

Assuming the monotonicity in Theorem D, the rigidity part follows by Lemma 4.3 and Theorem 2.1. For $p \in X \in \operatorname{Alex}^{n}(\kappa)$, let $A_{R}^{r}(p)$ (or briefly $A_{R}^{r}$ ) denote the annulus $\{x \in X: r<|p x| \leq R\}, 0 \leq r<R$, and let $A_{R}^{r}\left(\Sigma_{p}\right)$ (or briefly $\widetilde{A}_{R}^{r}$ ) denote the corresponding annulus in $C_{\kappa}\left(\Sigma_{p}\right)$. Let $B_{r}$ denote $A_{r}^{0}$ and let $\widetilde{B}_{r}$ denote $\widetilde{A}_{r}^{0}$. Let's recall the following two lemmas.

Lemma 4.1 [Li 2010, Lemma 2.1]. Let $\Sigma \in \operatorname{Alex}^{n-1}(1)$ and $0<r \leq \pi / \sqrt{\kappa}$. Then

$$
\operatorname{vol} C_{\kappa}^{r}(\Sigma)=\operatorname{vol} \Sigma \int_{0}^{r} \operatorname{sn}_{\kappa}^{n-1}(t) d t .
$$

Lemma 4.2 [Li 2010, Theorem B]. Let $U$ be an open subset in $X \in \operatorname{Alex}^{n}(\kappa)$. Then there is a constant $c(n)$ depending only on $n$ such that

$$
V_{r_{n}}(\bar{U})=V_{r_{n}}(U)=c(n) \operatorname{Haus}_{n}(U)=c(n) \operatorname{Haus}_{n}(\bar{U}),
$$

where $V_{r_{n}}$ and Haus $_{n}$ represent the $n$-dimensional rough volume and Hausdorff measure, respectively.

Lemma 4.3. If the monotonicity in Theorem B holds,

$$
\frac{\operatorname{vol} B_{r}}{\operatorname{vol} \widetilde{B}_{r}}=\frac{\operatorname{vol} B_{R}}{\operatorname{vol} \widetilde{B}_{R}}
$$

for some $0<r<R(R \leq \pi / \sqrt{\kappa}$ for $\kappa>0)$ if and only if $\operatorname{vol} B_{R}=\operatorname{vol} \widetilde{B}_{R}$.

Proof. Assume vol $B_{R}=\operatorname{vol} \widetilde{B}_{R}$. The desired equation follows by the monotonicity:

$$
1=\frac{\operatorname{vol} B_{R}}{\operatorname{vol} \widetilde{B}_{R}} \leq \frac{\operatorname{vol} B_{r}}{\operatorname{vol} \widetilde{B}_{r}} \leq \lim _{r \geq t \rightarrow 0} \frac{\operatorname{vol} B_{t}}{\operatorname{vol} \widetilde{B}_{t}}=1
$$

Assume

$$
\frac{\operatorname{vol} B_{r}}{\operatorname{vol} \widetilde{B}_{r}}=\frac{\operatorname{vol} B_{R}}{\operatorname{vol} \widetilde{B}_{R}}
$$


for some $0<r<R$. Then for any $t<r$,

$$
\frac{\operatorname{vol} B_{t}}{\operatorname{vol} A_{R}^{r}}+\frac{\operatorname{vol} A_{R}^{t}}{\operatorname{vol} A_{R}^{r}}=\frac{\operatorname{vol} B_{R}}{\operatorname{vol} A_{R}^{r}}=\frac{\operatorname{vol} \widetilde{B}_{R}}{\operatorname{vol} \widetilde{A}_{R}^{r}}=\frac{\operatorname{vol} \widetilde{B}_{t}}{\operatorname{vol} \widetilde{A}_{R}^{r}}+\frac{\operatorname{vol} \widetilde{A}_{R}^{t}}{\operatorname{vol} \widetilde{A}_{R}^{r}} .
$$

By the monotonicity, we have

$$
\frac{\operatorname{vol} A_{R}^{t}}{\operatorname{vol} A_{R}^{r}} \geq \frac{\operatorname{vol} \widetilde{A}_{R}^{t}}{\operatorname{vol} \widetilde{A}_{R}^{r}}
$$

Also,

$$
\frac{\operatorname{vol} B_{t}}{\operatorname{vol} A_{R}^{r}}=\frac{\operatorname{vol} B_{t}}{\operatorname{vol} A_{r}^{t}} \cdot \frac{\operatorname{vol} A_{r}^{t}}{\operatorname{vol} A_{R}^{r}} \geq \frac{\operatorname{vol} \widetilde{B}_{t}}{\operatorname{vol} \widetilde{A}_{r}^{t}} \cdot \frac{\operatorname{vol} \widetilde{A}_{r}^{t}}{\operatorname{vol} \widetilde{A}_{R}^{r}}=\frac{\operatorname{vol} \widetilde{B}_{t}}{\operatorname{vol} \widetilde{A}_{R}^{r}} .
$$

Consequently

$$
\frac{\operatorname{vol} B_{t}}{\operatorname{vol} A_{R}^{r}}=\frac{\operatorname{vol} \widetilde{B}_{t}}{\operatorname{vol} \widetilde{A}_{R}^{r}}, \quad \text { orequivalently, } \quad \frac{\operatorname{vol} B_{t}}{\operatorname{vol} \widetilde{B}_{t}}=\frac{\operatorname{vol} A_{R}^{r}}{\operatorname{vol} \widetilde{A}_{R}^{r}} .
$$

Letting $t \rightarrow 0$, we get vol $A_{R}^{r}=\operatorname{vol} \widetilde{A}_{R}^{r}$. Thus

$$
1 \geq \frac{\operatorname{vol} B_{R}}{\operatorname{vol} \widetilde{B}_{R}} \geq \frac{\operatorname{vol} A_{R}^{r}}{\operatorname{vol} \widetilde{A}_{R}^{r}}=1 .
$$

Now it remains to show the monotonicity in Theorem D. We take an elementary approach by expressing the monotonicity as a form of "Riemann sum" (see (4-5)) and using the Toponogov triangle comparison to bound each term in terms of the desired form (see Corollary 4.6). To achieve this goal, we choose a special infinite partition (see (4-5) and (4-6)).

We start the proof of Theorem $\mathrm{D}$ by deriving an equivalent form of the monotonicity. For $0 \leq R_{1}<R_{2}<R_{3}(<\pi / \sqrt{\kappa}$ when $\kappa>0)$, and $p \in X$, by Lemma 4.1, the monotonicity has the following integral form:

$$
\frac{\operatorname{vol} A_{R_{3}}^{R_{1}}}{\operatorname{vol} A_{R_{2}}^{R_{1}}} \leq \frac{\int_{R_{1}}^{R_{3}} \operatorname{sn}_{\kappa}^{n-1}(t) d t}{\int_{R_{1}}^{R_{2}} \operatorname{sn}_{\kappa}^{n-1}(t) d t},
$$

which is equivalent to

$$
I_{1}=\log \left[\frac{\operatorname{vol} A_{R_{3}}^{R_{1}}}{\operatorname{vol} A_{R_{2}}^{R_{1}}}\right] \leq \log \left[\frac{\int_{R_{1}}^{R_{3}} \operatorname{sn}_{\kappa}^{n-1}(t) d t}{\int_{R_{1}}^{R_{2}} \operatorname{sn}_{\kappa}^{n-1}(t) d t}\right]=I_{2} .
$$

Fixing a small $\delta>0$, let $m=\left[\left(R_{3}-R_{2}\right) / \delta\right]+1, \Delta=\left(R_{3}-R_{2}\right) / m \approx \delta$, and $r_{j}=R_{2}+j \Delta, 0 \leq j \leq m$. Then

$$
A_{R_{2}}^{R_{1}}=A_{r_{0}}^{R_{1}} \subset A_{r_{1}}^{R_{1}} \subset \cdots \subset A_{r_{m}}^{R_{1}}=A_{R_{3}}^{R_{1}} .
$$


Using the Taylor expansion $\log (1 / x)=1-x+O\left((1-x)^{2}\right)$, we may rewrite the left hand side of (4-1) as

$$
\begin{aligned}
I_{1} & =\sum_{j=1}^{m} \log \frac{\operatorname{vol} A_{R_{1}}^{r_{j}}}{\operatorname{vol} A_{R_{1}}^{r_{j-1}}}=\sum_{j=1}^{m}\left[\left(1-\frac{\operatorname{vol} A_{R_{1}}^{r_{j-1}}}{\operatorname{vol} A_{R_{1}}^{r_{j}}}\right)+O\left(\delta^{2}\right)\right] \\
& =\sum_{j=1}^{m} \frac{\operatorname{vol} A_{r_{j-1}}^{r_{j}}}{\operatorname{vol} A_{R_{1}}^{r_{j}}}+O(\delta) .
\end{aligned}
$$

Let $\phi(r)=\int_{R_{1}}^{r} \mathrm{sn}_{\kappa}^{n-1}(t) d t$. Then the right hand side of (4-1) can be written as

$$
\begin{aligned}
I_{2} & =\log \frac{\phi\left(R_{3}\right)}{\phi\left(R_{2}\right)}=\int_{R_{2}}^{R_{3}} \frac{\phi^{\prime}(t)}{\phi(t)} d t \\
& =\sum_{j=1}^{m} \frac{\phi^{\prime}\left(r_{j}\right)}{\phi\left(r_{j}\right)} \delta+\tau(\delta) \\
& =\sum_{j=1}^{m} \frac{\delta \mathrm{sn}_{\kappa}^{n-1}\left(r_{j}\right)}{\int_{R_{1}}^{r_{j}} \operatorname{sn}_{\kappa}^{n-1}(t) d t}+\tau(\delta) .
\end{aligned}
$$

Comparing (4-1) to (4-2) and (4-3), it's sufficient to show

$$
\frac{\operatorname{vol} A_{r_{j-1}}^{r_{j}}}{\operatorname{vol} A_{R_{1}}^{r_{j}}} \leq \frac{\delta \mathrm{sn}_{\kappa}^{n-1}\left(r_{j}\right)}{\int_{R_{1}}^{r_{j}} \operatorname{sn}_{\kappa}^{n-1}(t) d t} .
$$

We further divide $A_{R_{1}}^{r_{j}}$ into thinner annuli: given a monotonic sequence

$$
\left\{a_{i}\right\}_{i=1}^{\infty} \subset[0,1]
$$

such that $a_{j} \rightarrow 0,\left\{a_{i} r_{j}\right\}_{i=1}^{\infty}$ is an infinite partition for [0, $\left.r_{j}\right]$, and (4-4) is equivalent to

$$
\frac{\operatorname{vol} A_{R_{1}}^{r_{j}}}{\operatorname{vol} A_{r_{j-1}}^{r_{j}}}=\sum_{i=1}^{\infty} \frac{\operatorname{vol} A_{a_{i} r_{j}}^{a_{i+1} r_{j}}}{\operatorname{vol} A_{r_{j-1}}^{r_{j}}} \geq \frac{\int_{R_{1}}^{r_{j}} \operatorname{sn}_{\kappa}^{n-1}(t) d t}{\delta \operatorname{sn}_{\kappa}^{n-1}\left(r_{j}\right)} .
$$

To show (4-5), we need an estimate for vol $A_{a_{i} r_{j}}^{a_{i+1} r_{j}} / \operatorname{vol} A_{r_{j-1}}^{r_{j}}$ from below (see Corollary 4.6). Assume $\delta$ is so small that $R-\delta>0$ and $r-\lambda \delta>0$. Let $x \in A_{R-\delta}^{R}$. We define a map, $\phi: A_{R-\delta}^{R} \rightarrow A_{r-\lambda \delta}^{r}$, where $f(x)$ is the point on a minimal geodesic $[p x]$ (if not unique, we pick one of them) such that

$$
|p f(x)|=r-\lambda(R-|p x|) .
$$

Because a geodesic in $X$ does not branch, $\phi$ is well-defined and is injective.

In the proof of Theorem $\mathrm{D}$, the following is a main technical lemma, which asserts that $\phi$ behaves like a bi-Lipschitz function. 
Lemma 4.4. Let $\delta>0$ be sufficiently small, $\lambda=\left(\operatorname{sn}_{\kappa} r /\left(\mathrm{sn}_{\kappa} R\right)\right)$, and

$$
\phi: \widetilde{A}_{R-\delta}^{R} \rightarrow \widetilde{A}_{r-\lambda \delta}^{r}
$$

be defined as above. Then

$$
c(\kappa, \delta) \lambda \leq \frac{\operatorname{sn}_{\kappa}(|\phi(x) \phi(y)| / 2)}{\operatorname{sn}_{\kappa}(|x y| / 2)} \leq c(\kappa, \delta)^{-1} \lambda,
$$

where

$$
c(\kappa, \delta)=\left\{\begin{array}{ll}
1 & \kappa=0, \\
1-\frac{2 \delta}{\operatorname{sn}_{\kappa} R+\delta} & \kappa>0, \\
1-\delta \frac{\cosh h_{\kappa} R}{R} & \kappa<0
\end{array} .\right.
$$

Because the proof of Lemma 4.4 is technical and somewhat tedious, we will delay it to the end of this section.

Lemma 4.5. Let $U$ and $V$ be two open subsets of $X \in \operatorname{Alex}^{n}(\kappa)$, and let $\phi: V \rightarrow U$ be an injection. If $\phi$ satisfies $\operatorname{sn}_{\kappa}(|\phi(x) \phi(y)| / 2) \geq c \operatorname{sn}_{\kappa}(|x y| / 2)$ for any $x, y \in V$, $\operatorname{vol} U \geq c^{n}$ vol $V$, where $c$ is a constant.

Proof. By Lemma 4.2, it suffices to prove for rough volume. Recall that the $n$ dimensional rough volume of a subset $V$ is

$$
V_{r_{n}}(V)=\lim _{\epsilon \rightarrow 0} \epsilon^{n} \beta_{V}(\epsilon),
$$

where $\beta_{V}(\epsilon)$ denotes the number of points in an $\epsilon$-net $\left\{x_{i}\right\}$ on $V$.

By the assumption, $\left\{\phi\left(x_{i}\right)\right\}$ is a $2 \operatorname{sn}_{\kappa}^{-1}\left(c \operatorname{sn}_{\kappa}(\epsilon / 2)\right)$-net in $U$. We get

$$
\beta_{U}\left(2 \operatorname{sn}_{\kappa}^{-1}\left(c \operatorname{sn}_{\kappa} \frac{\epsilon}{2}\right)\right) \geq \beta_{V}(\epsilon)
$$

or in another form,

$$
\frac{\epsilon^{n}}{\left(2 \operatorname{sn}_{\kappa}^{-1}\left(c \operatorname{sn}_{\kappa}(\epsilon / 2)\right)\right)^{n}}\left(2 \operatorname{sn}_{\kappa}^{-1}\left(c \operatorname{sn}_{\kappa} \frac{\epsilon}{2}\right)\right)^{n} \beta_{U}\left(2 \operatorname{sn}_{\kappa}^{-1}\left(\operatorname{sn}_{\kappa} \frac{\epsilon}{2}\right)\right) \geq \epsilon^{n} \beta_{V}(\epsilon) .
$$

Letting $\epsilon \rightarrow 0$, we get $\left(1 / c^{n}\right) V_{r_{n}}(U) \geq V_{r_{n}}(V)$.

Corollary 4.6. Let $p \in X \in \operatorname{Alex}^{n}(\kappa), \delta>0$ small. Then

$$
\frac{\operatorname{vol} A_{r-\lambda \delta}^{r}}{\operatorname{vol} A_{R-\delta}^{R}} \geq(1-\tau(\delta))\left(\frac{\operatorname{sn}_{\kappa} r}{\operatorname{sn}_{\kappa} R}\right)^{n} .
$$

Proof. Consider the map $\phi: A_{R-\delta}^{R} \rightarrow A_{r-\lambda \delta}^{r}$ and $\tilde{\phi}: \widetilde{A}_{R-\delta}^{R} \rightarrow \widetilde{A}_{r-\lambda \delta}^{r}$ defined as above. For any $x, y \in A_{R-\delta}^{R}$, take two points $\tilde{x}, \tilde{y} \in C_{\kappa}\left(\Sigma_{p}\right)$ such that $|\tilde{o} \tilde{x}|=|p x|$, 
$|\tilde{o} \tilde{y}|=|p y|$, and $|\tilde{x} \tilde{y}|=|x y|$. By condition B (see [Burago et al. 1992]), it's easy to see that $|f(x) f(y)| \geq|\tilde{\phi}(\tilde{x}) \tilde{\phi}(\tilde{y})|$. Thus, by Lemma 4.4, we have

$$
\operatorname{sn}_{\kappa} \frac{|f(x) f(y)|}{2} \geq \operatorname{sn}_{\kappa} \frac{|\tilde{\phi}(\tilde{x}) \tilde{\phi}(\tilde{y})|}{2} \geq(1-\tau(\delta)) \operatorname{sn}_{\kappa} \frac{|\tilde{x} \tilde{y}|}{2}=(1-\tau(\delta)) \operatorname{sn}_{\kappa} \frac{|x y|}{2} .
$$

Then we get the desired estimate by Lemma 4.5.

Proof of the monotonicity in Theorem D. Continuing from the earlier discussion, the proof reduces to verifying (4-5). We now take $\delta>0$ sufficiently small, and choose the sequence $\left\{a_{i}\right\}_{i=0}^{\infty}$ as

$$
a_{0}=1, \quad a_{i+1}=a_{i}-\frac{\operatorname{sn}_{\kappa}\left(a_{i} r_{j}\right)}{r_{j} \operatorname{sn}_{\kappa} r_{j}} \delta, \quad i=0,1, \ldots
$$

Then

$$
0<a_{i+1} \leq \begin{cases}\left(1-\frac{\delta}{r_{j}}\right) a_{i}, & \text { if } \kappa \geq 0, \\ \left(1-\frac{\delta}{\operatorname{sn}_{\kappa} r_{j}}\right) a_{i}, & \text { if } \kappa<0,\end{cases}
$$

and thus $a_{i} \rightarrow 0$ and is monotonically decreasing. For each $0 \leq i<\infty$ and $0 \leq j \leq m$, consider the map, $\phi: A_{r_{j}-\delta}^{r_{j}} \rightarrow A_{a_{i} r_{j}-\lambda_{i} \delta}^{a_{i} r_{j}}=A_{a_{i+1} r_{j}}^{a_{i} r_{j}}$, with $\lambda_{i}=\operatorname{sn}_{\kappa}\left(a_{i} r_{j}\right) / \mathrm{sn}_{\kappa}\left(r_{j}\right)$. By Corollary 4.6, we obtain that

$$
\frac{\left.\operatorname{vol} A_{a_{i+1} r_{j}}^{a_{i} r_{j}}\right)}{\operatorname{vol} A_{r_{j}-\delta}^{r_{j}}} \geq(1-\tau(\delta))\left(\frac{\operatorname{sn}_{\kappa}\left(a_{i} r_{j}\right)}{\operatorname{sn}_{\kappa} r_{j}}\right)^{n} .
$$

Observe that for $\delta \rightarrow 0,\left\{a_{i}\right\}$ will become more dense, and thus we can take $N_{\delta}>0$ such that $a_{N_{\delta}} r_{j} \geq R_{1}$ and $a_{N_{\delta}} r_{j} \rightarrow R_{1}$ as $\delta \rightarrow 0$. Summing up for $i=0,1, \ldots, N_{\delta}$, we get

$$
\begin{aligned}
\frac{\operatorname{vol} A_{r_{j}}^{R_{1}}}{\operatorname{vol} A_{r_{j}-\delta}^{r_{j}}} & \geq \frac{\left.\sum_{i=0}^{N_{\delta}} \operatorname{vol} A_{a_{i+1} r_{j}}^{a_{i} r_{j}}\right)}{\operatorname{vol} A_{r_{j}-\delta}^{r_{j}}} \\
& \geq \sum_{i=0}^{N_{\delta}}(1-\tau(\delta))\left(\frac{\operatorname{sn}_{\kappa}\left(a_{i} r_{j}\right)}{\operatorname{sn}_{\kappa} r_{j}}\right)^{n} \\
& \geq(1-\tau(\delta)) \frac{1}{\delta \mathrm{sn}_{\kappa}^{n-1}\left(r_{j}\right)} \sum_{i=0}^{N_{\delta}} \operatorname{sn}_{\kappa}^{n-1}\left(a_{i} r_{j}\right) \frac{\delta \operatorname{sn}_{\kappa}\left(a_{i} r_{j}\right)}{\operatorname{sn}_{\kappa} r_{j}} \\
& =(1-\tau(\delta)) \frac{1}{\delta \mathrm{sn}_{\kappa}^{n-1}\left(r_{j}\right)}\left(\int_{R_{1}}^{r_{j}} \operatorname{sn}_{\kappa}^{n-1}(t) d t+\tau(\delta)\right) \\
& =(1-\tau(\delta)) \frac{\int_{R_{1}}^{r_{j}} \operatorname{sn}_{\kappa}^{n-1}(t) d t}{\delta \operatorname{sn}_{\kappa}^{n-1}\left(r_{j}\right)}
\end{aligned}
$$


or the following equivalent form:

$$
\frac{\operatorname{vol} A_{r_{j}-\delta}^{r_{j}}}{\operatorname{vol} A_{r_{j}}^{R_{1}}} \leq(1+\tau(\delta)) \frac{\delta \mathrm{sn}_{\kappa}^{n-1}\left(r_{j}\right)}{\int_{R_{1}}^{r_{j}} \operatorname{sn}_{\kappa}^{n-1}(t) d t} .
$$

Summing up for all $j$ and together with (4-2) and (4-3), we get

$$
I_{1}+O(\delta) \leq(1+\tau(\delta)) I_{2}+\tau(\delta) .
$$

Letting $\delta \rightarrow 0$, we get the desired inequality.

The rest of this section is devoted to a proof of Lemma 4.4. The following are some properties used in the proof.

Lemma 4.7. (1) For $\lambda \in[0,1]$ and $x \in[0, \pi]$, $\sin \lambda x \geq \lambda \sin x$.

(2) For $\lambda \in[0,1]$ and $x \geq 0, \sinh \lambda x \leq \lambda \sinh x$.

(3) For $\lambda \geq 0$ and $x \geq 0, \sin \lambda x /(\lambda \sin x) \geq 1-(\lambda x)^{2} / 6$.

(4) For $\lambda \geq 0$ and $x \geq 0, \sinh \lambda x /(\lambda \sinh x) \geq x / \sinh x \geq 1-x$.

(5) Let $\triangle$ pab be a triangle in $S_{\kappa}^{2}$. The cosine law can be written as

$$
s n_{\kappa}^{2} \frac{|a b|}{2}=\operatorname{sn}_{\kappa}^{2} \frac{|p a|-|p b|}{2}+\sin ^{2} \frac{\measuredangle a p b}{2} \operatorname{sn}_{\kappa}|p a| \operatorname{sn}_{\kappa}|p b| .
$$

Proof.

(1) Let $h(x)=\sin \lambda x-\lambda \sin x$. Then

$$
h^{\prime}(x)=\lambda \cos \lambda x-\lambda \cos x=\lambda(\cos \lambda x-\cos x) \geq 0,
$$

since $0 \leq \lambda x \leq x \leq \pi$.

(2) Let $h(x)=\sinh \lambda x-\lambda \sinh x$. Then

$$
h^{\prime}(x)=\lambda \cosh \lambda x-\lambda \cosh x=\lambda(\cosh \lambda x-\cosh x) \leq 0,
$$

since $0 \leq \lambda x \leq x$.

(3) For $x>0$, one can show that $x \geq \sin x \geq x-x^{3} / 6$. Then

$$
\frac{\sin \lambda x}{\lambda \sin x} \geq \frac{\lambda x-(\lambda x)^{3} / 6}{\lambda x}=1-(\lambda x)^{2} / 6 .
$$

(4) The first equality is easy to see through $\sinh \lambda x \geq \lambda x$. Obviously, the second equality is true for $x \geq 1$. For $0<x<1$,

$$
\sinh x=x+\frac{x^{3}}{6}+\cdots \leq x\left(1+x+x^{2}+\cdots\right)=\frac{x}{1-x} .
$$

(5) Follows by trigonometric metric identities.

Proof of Lemma 4.4. By scaling, we only need to check for $\kappa=1,-1$ and $\kappa=0$. 
Case $1(\kappa=1)$. Noting that

$$
\frac{\left|p x^{\prime}\right|-\left|p y^{\prime}\right|}{|p x|-|p y|}=\frac{\lambda(|p x|-|p y|)}{|p x|-|p y|}=\lambda
$$

by Lemma 4.7(3) and $0 \leq|| p x|-| p y|| \leq \delta<\frac{1}{2} \sin R$, we have

$$
\begin{aligned}
\sin \frac{|| p x^{\prime}|-| p y^{\prime}||}{2} & =\sin \left(\lambda \frac{|| p x|-| p y||}{2}\right) \\
& \geq\left(1-\frac{(\lambda \delta)^{2}}{6}\right) \lambda \sin \frac{|| p x|-| p y||}{2} \\
& \geq\left(1-\frac{\delta^{2}}{6 \sin ^{2} R}\right) \lambda \sin \frac{|| p x|-| p y||}{2} \\
& \geq\left(1-\frac{2 \delta}{\sin R+\delta}\right) \lambda \sin \frac{|| p x|-| p y||}{2} \\
& =\tau_{1} \lambda \sin \frac{|| p x|-| p y||}{2} .
\end{aligned}
$$

Thus

$$
\tau_{1} \lambda \leq \frac{\sin \left(|| p x^{\prime}|-| p y^{\prime}|| / 2\right)}{\sin (|| p x|-| p y|| / 2)} \leq \frac{\lambda|| p x|-| p y|| / 2}{\sin (|| p x|-| p y|| / 2)} \leq \lambda \frac{\delta}{\sin \delta} \leq \tau_{1}^{-1} \lambda .
$$

For any $x \in \widetilde{A}_{R-\delta}^{R}$, by Lemma 4.7(1), we have

$\sin \left|p x^{\prime}\right| \geq \frac{\left|p x^{\prime}\right|}{r} \sin r \geq \frac{r-\lambda \delta}{r} \sin r=\frac{r-(\sin r / \sin R) \delta}{r} \sin r \geq\left(1-\frac{\delta}{\sin R}\right) \sin r$,

which, together with

$$
\sin \left|p x^{\prime}\right|-\sin r=2 \sin \frac{\left|p x^{\prime}\right|-r}{2} \cos \frac{\left|p x^{\prime}\right|+r}{2} \leq r-\left|p x^{\prime}\right| \leq \lambda \delta,
$$

gives us

$$
\left(1-\frac{\delta}{\sin R}\right) \sin r \leq \sin \left|p x^{\prime}\right| \leq \sin r+\lambda \delta=\left(1+\frac{\delta}{\sin R}\right) \sin r .
$$

Similarly,

$$
\sin |p x| \geq \frac{|p x|}{R} \sin R \geq \frac{R-\delta}{R} \sin R \geq\left(1-\frac{\delta}{\sin R}\right) \sin R
$$

and

$$
\sin |p x|-\sin R=2 \sin \frac{|p x|-R}{2} \cos \frac{|p x|+R}{2} \leq R-|p x| \leq \delta,
$$

hence

$$
\left(1-\frac{\delta}{\sin R}\right) \sin R \leq \sin |p x| \leq \sin R+\delta=\left(1+\frac{\delta}{\sin R}\right) \sin R .
$$


So

$$
c_{1} \frac{\sin r}{\sin R} \leq \frac{\sin \left|p x^{\prime}\right|}{\sin |p x|} \leq c_{1}^{-1} \frac{\sin r}{\sin R} .
$$

Let $\theta=\measuredangle x p y$. Since $|x y| / 2 \leq \pi / 2$, by the cosine law and inequalities (4-7), (4-8),

$$
\begin{aligned}
c_{1}^{2} \lambda^{2} & \leq \frac{\sin ^{2}\left(\left|x^{\prime} y^{\prime}\right| / 2\right)}{\sin ^{2}(|x y| / 2)} \\
& =\frac{\sin ^{2}\left(\left(\left|p x^{\prime}\right|-\left|p y^{\prime}\right|\right) / 2\right)+\sin ^{2}(\theta / 2) \sin \left|p x^{\prime}\right| \sin \left|p y^{\prime}\right|}{\sin ^{2}((|p x|-|p y|) / 2)+\sin ^{2}(\theta / 2) \sin |p x| \sin |p y|} \leq c_{1}^{-2} \lambda^{2} .
\end{aligned}
$$

Case $2(\kappa=-1)$. By Lemma 4.7(2),

$$
\lambda \delta=\frac{\sinh r}{\sinh R} \cdot \frac{R}{\cosh R}<\frac{r}{R} R=r,
$$

which, together with Lemma 4.7(4), gives

$$
\lambda \geq \frac{\sinh \left(|| p x^{\prime}|-| p y^{\prime}|| / 2\right)}{\sinh (|| p x|-| p y|| / 2)}=\frac{\sinh (\lambda|| p x|-| p y|| / 2)}{\sinh (|| p x|-| p y|| / 2)} \geq(1-\delta) \lambda \geq c_{-1} \lambda
$$

since

$$
\frac{\cosh R}{R} \geq \frac{1+R^{2} / 2}{R}>1 .
$$

If $\delta<R / \cosh R<R$, we have

$$
\frac{\lambda \delta}{2 r}<\frac{r}{R} \cdot \frac{\delta}{2 r}=\frac{\delta}{2 R}<1 .
$$

Hence we can apply Lemma 4.7(2) with $\lambda=(\sinh r) / \sinh R \leq r / R$, to get

$$
\frac{\sinh r-\sinh (r-\lambda \delta)}{\sinh r} \leq \frac{2 \sinh (\lambda \delta / 2) \cosh r}{\sinh r} \leq \frac{\lambda \delta}{r} \cosh r \leq \frac{\delta \cosh R}{R} .
$$

Thus

$$
\sinh (r-\lambda \delta) \geq\left(1-\delta \frac{\cosh R}{R}\right) \sinh r .
$$

For $x^{\prime} \in \widetilde{A}_{r-\lambda \delta}^{r}$,

$$
\left(1-\delta \frac{\cosh R}{R}\right) \sinh r \leq \sinh (r-\lambda \delta) \leq \sinh \left|p x^{\prime}\right| \leq \sinh r .
$$

For $x \in \widetilde{A}_{R-\lambda \delta}^{R}$,

$$
\frac{\sinh R-\sinh (R-\delta)}{\sinh R} \leq \frac{2 \sinh (\delta / 2) \cosh R}{\sinh R} \leq \frac{\delta \cosh R}{R},
$$

and

$$
\left(1-\delta \frac{\cosh R}{R}\right) \sinh R \leq \sinh (R-\lambda \delta) \leq \sinh |p x| \leq \sinh R
$$


Then

$$
c_{-1} \frac{\sinh r}{\sinh R} \leq \frac{\sinh \left|p x^{\prime}\right|}{\sinh |p x|} \leq c_{-1}^{-1} \frac{\sinh r}{\sinh R} .
$$

By inequalities (4-9), (4-10), and the cosine law, we get

$$
\begin{aligned}
c_{-1}^{2} \lambda^{2} & \leq \frac{\sinh ^{2}\left(\left|x^{\prime} y^{\prime}\right| / 2\right)}{\sinh ^{2}(|x y| / 2)} \\
& =\frac{\sinh ^{2}\left(\left(\left|p x^{\prime}\right|-\left|p y^{\prime}\right|\right) / 2\right)+\sin ^{2}(\theta / 2) \sinh \left|p x^{\prime}\right| \sinh \left|p y^{\prime}\right|}{\sinh ^{2}((|p x|-|p y|) / 2)+\sin ^{2}(\theta / 2) \sinh |p x| \sinh |p y|} \leq c_{-1}^{-2} \lambda^{2} .
\end{aligned}
$$

Case $3(\kappa=0)$. This is straightforward.

\section{References}

[Bredon 1972] G. E. Bredon, Introduction to compact transformation groups, Pure and Applied Mathematics 46, Academic, New York, 1972. MR 54 \#1265 Zbl 0246.57017

[Burago et al. 1992] Y. Burago, M. Gromov, and G. Perelman, "A. D. Aleksandrov spaces with curvatures bounded below”, Uspekhi Mat. Nauk 47:2(284) (1992), 3-51. In Russian; translated in Russian Math. Surveys 47:2 (1992), 1-58. MR 93m:53035 Zbl 0802.53018

[Burago et al. 2001] D. Burago, Y. Burago, and S. Ivanov, A course in metric geometry, Graduate Studies in Mathematics 33, American Mathematical Society, Providence, RI, 2001. MR 1835418 (2002e:53053) Zbl 0981.51016

[Grove and Petersen 1992] K. Grove and P. Petersen, V, "Volume comparison à la Aleksandrov", Acta Math. 169:1-2 (1992), 131-151. MR 93j:53057

[Kapovitch 2002] V. Kapovitch, "Regularity of limits of noncollapsing sequences of manifolds", Geom. Funct. Anal. 12:1 (2002), 121-137. MR 2003b:53043 Zbl 1013.53046

[Kapovitch 2007] V. Kapovitch, "Perelman's stability theorem", pp. 103-136 in Metric and comparison geometry, edited by J. Cheeger and K. Grove, Surv. Differ. Geom. 11, International Press, Somerville, MA, 2007. MR 2009g:53057 Zbl 1151.53038

[Li 2010] N. Li, Controlled geometry via volumes on Alexandrov spaces, thesis, Rutgers University, 2010, Available at http://wwwlib.umi.com/dissertations/fullcit/3434721. MR 2827253

[Liu and Shen 1994] Z.-d. Liu and Z. M. Shen, "On the Betti numbers of Alexandrov spaces", Ann. Global Anal. Geom. 12:2 (1994), 123-133. MR 95b:53055 Zbl 0824.53041

[Perelman 1991] G. Perelman, "Alexandrov spaces with curvatures bounded from below, II", preprint, 1991.

[Petrunin 1997] A. Petrunin, "Applications of quasigeodesics and gradient curves", pp. 203-219 in Comparison geometry (Berkeley, CA, 1993-94), edited by K. Grove and P. Petersen, Math. Sci. Res. Inst. Publ. 30, Cambridge University Press, 1997. MR 98m:53061 Zbl 0892.53026

[Petrunin 1998] A. Petrunin, "Parallel transportation for Alexandrov space with curvature bounded below”, Geom. Funct. Anal. 8:1 (1998), 123-148. MR 98j:53048 Zbl 0903.53045

[Petrunin 2007] A. Petrunin, "Semiconcave functions in Alexandrov's geometry", pp. 137-201 in Metric and comparison geometry, edited by J. Cheeger and K. Grove, Surv. Differ. Geom. 11, International Press, Somerville, MA, 2007. MR 2010a:53052 Zbl 1166.53001

[Shteingold 1994] S. Shteingold, "Volume comparison for Alexandrov spaces", Indiana Univ. Math. J. 43:4 (1994), 1349-1357. MR 96c:53065 Zbl 0833.53039 
[Wu 1997] J.-Y. Wu, "Topological regularity theorems for Alexandrov spaces", J. Math. Soc. Japan 49:4 (1997), 741-757. MR 1466363 (98h:53071) Zbl 0915.53021

Received December 10, 2011.

NAN LI

DEPARTMENT OF MATHEMATICS

UNIVERSITY OF NOTRE DAME

255 HURLEY HALL

NOTRE DAME, IN 46556

UNITED STATES

lilinanan@gmail.com

XiaOchun Rong

DEPARTMENT OF MATHEMATiCs

CAPITAL NORMAL UNIVERSITY

105 XI SAN HUAN BEI RD.

HAIDIAN DISTRICT

BEIJING 100048

CHINA

and

DEPARTMENT OF MATHEMATICS

RUTGERS UNIVERSITY

Hill Center for the Mathematical Sciences

110 FRELINGHUYSEN RD

PisCATAWAy, NEW JERSEY 08854

United STATES

rong@math.rutgers.edu 


\title{
PACIFIC JOURNAL OF MATHEMATICS
}

\author{
http://pacificmath.org \\ Founded in 1951 by \\ E. F. Beckenbach (1906-1982) and F. Wolf (1904-1989)
}

\section{EDITORS}

V. S. Varadarajan (Managing Editor)

Department of Mathematics

University of California

Los Angeles, CA 90095-1555

pacific@math.ucla.edu

Vyjayanthi Chari

Department of Mathematics

University of California

Riverside, CA 92521-0135

chari@math.ucr.edu

\section{Robert Finn}

Department of Mathematics Stanford University

Stanford, CA 94305-2125

finn@math.stanford.edu

Kefeng Liu

Department of Mathematics

University of California

Los Angeles, CA 90095-1555

liu@math.ucla.edu
Darren Long

Department of Mathematics

University of California

Santa Barbara, CA 93106-3080

long@math.ucsb.edu

Jiang-Hua Lu

Department of Mathematics

The University of Hong Kong

Pokfulam Rd., Hong Kong jhlu@maths.hku.hk

Alexander Merkurjev

Department of Mathematics

University of California

Los Angeles, CA 90095-1555

merkurev@math.ucla.edu
Sorin Popa

Department of Mathematics University of California

Los Angeles, CA 90095-1555 popa@math.ucla.edu

Jie Qing

Department of Mathematics

University of California

Santa Cruz, CA 95064

qing@cats.ucsc.edu

Jonathan Rogawski

Department of Mathematics

University of California

Los Angeles, CA 90095-1555

jonr@math.ucla.edu

\section{PRODUCTION}

pacific@math.berkeley.edu

\section{SUPPORTING INSTITUTIONS}

ACADEMIA SINICA, TAIPEI

CALIFORNIA INST. OF TECHNOLOGY INST. DE MATEMÁTICA PURA E APLICADA KEIO UNIVERSITY

MATH. SCIENCES RESEARCH INSTITUTE NEW MEXICO STATE UNIV.

OREGON STATE UNIV.

\author{
STANFORD UNIVERSITY \\ UNIV. OF BRITISH COLUMBIA \\ UNIV. OF CALIFORNIA, BERKELEY \\ UNIV. OF CALIFORNIA, DAVIS \\ UNIV. OF CALIFORNIA, LOS ANGELES \\ UNIV. OF CALIFORNIA, RIVERSIDE \\ UNIV. OF CALIFORNIA, SAN DIEGO \\ UNIV. OF CALIF., SANTA BARBARA
}

\author{
UNIV. OF CALIF., SANTA CRUZ \\ UNIV. OF MONTANA \\ UNIV. OF OREGON \\ UNIV. OF SOUTHERN CALIFORNIA \\ UNIV. OF UTAH \\ UNIV. OF WASHINGTON \\ WASHINGTON STATE UNIVERSITY
}

These supporting institutions contribute to the cost of publication of this Journal, but they are not owners or publishers and have no responsibility for its contents or policies.

See inside back cover or pacificmath.org for submission instructions.

The subscription price for 2012 is US \$420/year for the electronic version, and \$485/year for print and electronic.

Subscriptions, requests for back issues from the last three years and changes of subscribers address should be sent to Pacific Journal of Mathematics, P.O. Box 4163, Berkeley, CA 94704-0163, U.S.A. Prior back issues are obtainable from Periodicals Service Company, 11 Main Street, Germantown, NY 12526-5635. The Pacific Journal of Mathematics is indexed by Mathematical Reviews, Zentralblatt MATH, PASCAL CNRS Index, Referativnyi Zhurnal, Current Mathematical Publications and the Science Citation Index.

The Pacific Journal of Mathematics (ISSN 0030-8730) at the University of California, c/o Department of Mathematics, 969 Evans Hall, Berkeley, CA 94720-3840, is published monthly except July and August. Periodical rate postage paid at Berkeley, CA 94704, and additional mailing offices. POSTMASTER: send address changes to Pacific Journal of Mathematics, P.O. Box 4163, Berkeley, CA 94704-0163.

PJM peer review and production are managed by EditFLOW ${ }^{\mathrm{TM}}$ from Mathematical Sciences Publishers.

PUBLISHED BY PACIFIC JOURNAL OF MATHEMATICS

at the University of California, Berkeley 94720-3840

A NON-PROFIT CORPORATION

Typeset in LATEX

Copyright $(02012$ by Pacific Journal of Mathematics 


\section{PACIFIC JOURNAL OF MATHEMATICS}

Volume $259 \quad$ No. $2 \quad$ October 2012

Flag subdivisions and $\gamma$-vectors

257

Christos A. ATHANASIAdis

Rays and souls in von Mangoldt planes

279

IGOR BELEGRADEK, ERIC CHOI and NOBUHIRO INNAMI

Isoperimetric surfaces with boundary, II

ABRAHAM FRANDSEN, DONALD SAMPSON and NEIL

STEINBURG

Cyclic branched coverings of knots and quandle homology

YUICHI KABAYA

On a class of semihereditary crossed-product orders

JOHN S. KAUTA

An explicit formula for spherical curves with constant torsion

DEMETRE KAZARAS and IVAN STERLING

Comparing seminorms on homology

JEAN-FranÇOIS LAFONT and CHRISTOPHE PITTET

Relatively maximum volume rigidity in Alexandrov geometry

NAN LI and XIAOCHUN RONG

Properness, Cauchy indivisibility and the Weil completion of a group of 421 isometries

Antonios Manoussos and Polychronis Strantzalos

Theta lifts of strongly positive discrete series: the case of $(\tilde{\mathrm{Sp}}(n), O(V)) 445$

IVAN MATIĆ

Tunnel one, fibered links

MATt RATHBUN

Fusion symmetric spaces and subfactors

HANS WENZL 